\title{
Asymptotic Behavior of Solutions to the Coagulation-Fragmentation Equations. II. Weak Fragmentation
}

\author{
J. Carr ${ }^{1}$ and F. P. da Costa ${ }^{1.2}$
}

Received July 2, 1993

The discrete coagulation-fragmentation equations are a model for the kinetics of cluster growth in which clusters can coagulate via binary interactions to form larger clusters or fragment to form smaller ones. The assumptions made on the fragmentation coefficients have the physical interpretation that surface effects are important. Our results on the asymptotic behavior of solutions generalize the corresponding results of Ball, Carr, and Penrose for the Becker-Doring equation.

KEY WORDS: Clustering; coagulation; fragmentation; phase transition; asymptotic behavior.

\section{INTRODUCTION}

The discrete coagulation-fragmentation equations describe the kinetics of cluster growth in which clusters can coagulate, via binary interactions, to form larger clusters or fragment to form smaller ones. Denoting by $c_{j}(t) \geqslant 0$ the concentration of a cluster with $j$ particles ( $j$-cluster, for short), the equations are

$$
\dot{c}_{j}=\frac{1}{2} \sum_{k=1}^{j-1} W_{j-k, k}(c)-\sum_{k=1}^{\infty} W_{j, k}(c)
$$

for $j=1,2, \ldots$, where $W_{j, k}(c)=a_{j, k} c_{j} c_{k}-b_{j, k} c_{j+k}$, with $c=\left(c_{j}\right)$. The coagulation and the fragmentation rate coefficients, respectively $a_{j, k}$

Dedicated to Oliver Penrose on the occasion of his 65 th birthday

1 Department of Mathematics, Heriot-Watt University, Edinburgh EH14 4AS. Scotland, United Kingdom. E-mail: Jack (àcara.ma.hw.ac.uk.

${ }^{2}$ Present address: Instituto Superior Técnico, Departamento de Matemática, P-1096, Lisbon, Portugal. 
and $b_{j, k}$, are nonnegative constants, and are symmetric with respect to permutation of the subscripts.

From physical considerations, it is only relevant to consider solutions to (1.1) which are nonnegative and have finite density $\rho(t)=\sum_{j=1}^{\infty} j c_{j}(t)$. This motivates the study of solutions to (1.1) in the Banach space of finitedensity sequences

$$
X=\left\{c=\left(c_{j}\right):\|c\| \stackrel{\text { der }}{=} \sum_{j=1}^{\infty} j\left|c_{j}\right|<\infty\right\}
$$

Because each interaction preserves the number of particles, we expect the density $\rho(t)$ to be a conserved quantity. However, for some rate coefficients, $\rho(t)$ may not be constant. ${ }^{(5.7)}$ Throughout this paper we assume that

$$
a_{j, k} \leqslant K_{a}(j+k)
$$

for all $j, k \geqslant 1$, and some constant $K_{a}>0$. This condition ensures coagulation does not lead to the breakdown of density conservation. ${ }^{(2)}$ Equations (1.1), as well as some important special cases such as the Smoluchowski $\left(b_{j, k} \equiv 0\right.$ for all $\left.j, k\right)$ and the Becker-Döring equations $\left(a_{j, k}=b_{j, k}=0\right.$ if $\min \{j, k\}>1$ ), have been the focus of a number of mathematical papers in recent years (see, e.g., refs. $1-4$ and 6 ). In particular, results concerning existence, uniqueness, and density conservation of solutions of (1.1) were proved in ref. 2 for coagulation coefficients satisfying (1.3). Two classes of fragmentation coefficients have been identified, each leading to distinctive behavior of solutions.

In a recent paper, $\mathrm{Carr}^{(4)}$ considered the following "strong fragmentation" condition, namely that there exists a $\gamma>0$ such that for all $m \geqslant 0$, there is a constant $C(m)>0$ such that

$$
\sum_{j=1}^{[(r-1) / 2]} j^{m} b_{j, r-j} \geqslant C(m) r^{\gamma+m}
$$

for all $r \geqslant 3$, where $[x]$ denotes the integer part of $x$. The main result of ref. 4 concerns the $\mu^{\text {th }}$ moment $S_{\mu}(t)$ of a solution

$$
S_{\mu}(t)=\sum_{j=1}^{\infty} j^{\mu} c_{j}(t)
$$

If (1.3) and (1.4) hold, then if $c$ is a density-conserving solution, for every $\mu>0, S_{\mu}(t)<\infty$ for all $t>0$. Thus, for this class of solutions, strong fragmentation acts as a smoothing mechanism; in particular, orbits are precompact in $X$. It is essential to restrict the class of solutions when (1.4) holds, since in general, solutions are not unique (see examples in ref. 2). 
The precompactness of orbits of density-conserving solutions implies, in particular, that every such orbit has a nonempty $\omega$-limit set in $X$. With some additional assumptions, the most important of which is that a detailed balance condition holds, namely, the existence of a positive sequence $\left(Q_{j}\right)$ with $Q_{1}=1$ and such that, for all $j$ and $k$,

$$
a_{j, k} Q_{j} Q_{k}=b_{j, k} Q_{j+k}
$$

the $\omega$-limit set of any orbit can be proved to be a single equilibrium $c^{\rho_{0}}$ with density $\rho_{0}=\|c(0)\|$.

This behavior contrasts markedly with what happens for the BeckerDöring system ${ }^{(1,3,6)}$ for which one can have a behavior that can be physically interpreted as a dynamic phase transition: the existence of a critical density $\rho_{s} \in(0, \infty)$ such that orbits are precompact in $X$ if and only if $\rho_{0} \leqslant \rho_{s}$. Under convenient conditions on the coefficients, this compactness result implies that if $\rho_{0} \leqslant \rho_{s}$, then solutions converge strongly in $X$ to an equilibrium with density $\rho_{0}$. If $\rho_{0}>\rho_{s}$, solutions converge weak* but not strongly in $X$, to an equilibrium with density $\rho_{s}$ with the excess density $\rho_{0}-\rho_{s}$ being "transferred" to infinity. This behavior can be physically interpreted as a condensation phenomenon. ${ }^{(3)}$

In this paper we make use of a "weak fragmentation" condition that allow us to prove results similar to the ones described above for the Becker-Döring system. We say the weak fragmentation condition holds if there exists a constant $K_{f}>0$ such that for all $r>1$,

$$
\sum_{j=1}^{h(r)} j b_{j, r-j} \leqslant K_{f} r
$$

where $h(r)=[(r+1) / 2]$. With these conditions it was proved in ref. 2 that all solutions conserve density. Moreover, under a condition slightly stronger than (1.7), uniqueness was proved. ${ }^{(2)}$

Both classes of fragmentation coefficients, (1.4) and (1.7), are physically important. For example, in unbranched polymeric chains, for which the probability of breaking a bond between two monomeric units is independent of the sizes of both the original chain and the resulting ones, we have $b_{j, k} \equiv b=$ const and (1.4) is satisfies with $\gamma=1$. On the other hand, in cases where the surface energy of the cluster plays an important role, we can expect the fragmentation coefficients $b_{j, k}$ to be very small if both $j$ and $k$ are large. A very simple example of rate coefficients exhibiting this behavior is $b_{j . k}=(j k)^{-\beta}$, which satisfies (1.7) if $\beta>-1$. Another one is

$$
b_{j, k}=K(j+k) \exp \left\{\lambda\left[(j+k)^{\mu}-j^{\mu}-k^{\mu}\right]\right\}
$$


where $K, \lambda>0$ and $\mu \in(0,1)$ are constants. ${ }^{(2)}$ For the Becker-Döring equations in which all fragmentation coefficients are zero except $b_{r, 1}=b_{1, r}$, condition (1.7) holds if $b_{r .1} \leqslant$ const $\cdot r$.

This paper makes frequent use of results from ref. 2 , but can be read independently of ref. 4. It is organized as follows:

In Section 2 we state the basic hypotheses and definitions and recall some general results that will be needed afterward.

Section 3 deals with the behavior of the higher moments (1.5) of solutions to (1.1). It is shown that, if (1.7) holds, an initially $(t=0)$ infinite moment stays infinite for every finite time. Also in this section we prove that, although solutions do not become more regular, at least some solutions [ the admissible ones, obtained as limits of an appropriate finitedimensional truncation of (1.1)] do not become less regular either, i.e., if $c$ is an admissible solution of (1.1) and if the $\mu$-moment $S_{\mu}$ is initially finite, then it stays finite for all finite time.

In Section 4 we start the study of the asymptotic behavior of solutions by considering the simple cases of pure fragmentation $\left(a_{j, k} \equiv 0\right)$ and pure coagulation $\left(b_{j, k} \equiv 0\right)$. We prove that in both cases solutions converge to equilibria: for pure fragmentation, solutions converge to $\bar{c}_{j}=\rho_{0} \delta_{j, 1}$ strongly in $X$ as $t \rightarrow \infty$, where $\rho_{0}$ is the density of the initial data, and in the pure coagulation case solutions converge weak* to zero in $X$ at $t \rightarrow \infty$. In both cases the proofs rely on the monotonicity of the partial sums $p_{n}(t)=\sum_{j=1}^{n} j c_{j}(t)$ with respect to $t$.

For genuine coagulation-fragmentation equations a different method is needed since $p_{n}$ is no longer monotone. Assuming the detailed balance condition (1.6), we find that the sequences $c_{r}^{\rho}=Q_{r}\left(c_{1}^{\rho}\right)^{r}, r \geqslant 1$, with $c_{1}^{\rho}$ determined by the condition $\left\|c^{\rho}\right\|=\rho$, are equilibria of (1.1). In contrast with the strong fragmentation case, ${ }^{(4)}$ when the weak fragmentation condition (1.7) holds we can have a critical density $\rho_{s}$. In this case, no equilibrium $c^{\rho}$ exist if $\rho>\rho_{s}$, so we can expect similar results to those obtained for the Becker-Döring equations. In Section 5 we prove that the function

$$
V(c)=\sum_{j=1}^{\infty} c_{j}\left(\log \frac{c_{j}}{Q_{j}}-1\right)
$$

is a Lyapunov function for (1.1) provided $c_{j}(t)>0$ for all $j$ and $t$ and $\log Q_{j}$ satisfies a Lipschitz and a superadditivity condition.

Finally, in Section 6 we use the results of Section 5 to prove convergence to equilibrium for a solution with initial density $\rho_{0}$. We prove the weak* convergence of a solution with density $\rho_{0}$ to a unique equilibrium $c^{\rho}$ as $t \rightarrow \infty$, where the density of the limit equilibrium is $0 \leqslant \rho \leqslant$ $\min \left\{\rho_{0}, \rho_{s}\right\}$. Despite numerous attempts, it was not possible to establish in 
full generality the dynamic phase transition behavior proved to occur in the Becker-Döring equations, i.e., the distinction between strong convergence to $c^{\rho}$ for $\rho_{0} \leqslant \rho_{s}$ and weak* but not strong convergence if $\rho_{0}>\rho_{s}$. However, for the particular cases with $a_{j . k}=b_{j, k}=0$ iff $\min \{j, k\}>N$, for some integer constant $N$, we prove this result for rapidly decaying initial data.

\section{PRELIMINARIES}

\subsection{Definitions}

We study (1.1) in the set

$$
X^{+}=\left\{c \in X: c_{j} \geqslant 0 \text { for all } j \geqslant 1\right\}
$$

where $X$ is the Banach space of finite-density sequences defined by (1.2).

The following definition of solution, introduced in ref. 2 , is used.

Definition 1. Let $T \in(0, \infty]$. A solution $c=\left(c_{j}\right)$ of Eq. (1.1) on $[0, T)$ is a function $c:[0, T) \rightarrow X^{+}$such that:

1. Each $c_{j}:[0, T) \rightarrow \mathbf{R}$ is continuous and $\sup _{t \in[0, T)}\|c(t)\|<\infty$.

2. For all $j=1,2, \ldots$ and all $t \in[0, T)$,

$$
\int_{0}^{t} \sum_{k=1}^{\infty} a_{j, k} c_{k}(s) d s<\infty, \quad \int_{0}^{t} \sum_{k=1}^{\infty} b_{j, k} c_{j+k}(s) d s<\infty
$$

3. For all $j=1,2, \ldots$ and all $t \in[0, T)$,

$$
c_{j}(t)=c_{j}(0)+\int_{0}^{t}\left[\frac{1}{2} \sum_{k=1}^{j-1} W_{j-k, k}(c(s))-\sum_{k=1}^{\infty} W_{j, k}(c(s))\right] d s
$$

For $\rho \in[0, \infty)$ let $X^{\rho+}=\left\{c \in X^{+}:\|c\|=\rho\right\}$, and with $\rho \in(0, \infty)$ consider the closed ball of $X$ defined by

$$
B_{\rho}=\{c \in X:\|c\| \leqslant \rho\}
$$

The function $d(\alpha, \beta)=\sum_{j=1}^{\infty}\left|\alpha_{j}-\beta_{j}\right|$ is well defined for $\alpha, \beta \in B_{\rho}$, and $\left(B_{\rho}, d\right)$ is a metric space; furthermore, $B_{\rho}^{+}=B_{\rho} \cap X^{+}$with the metric $d(\cdot, \cdot)$ is a closed metric subspace of $\left(B_{\rho}, d\right)$.

We will use the notions of strong (norm) convergence in $X$ as well as that of weak convergence: a sequence $c^{(n)}$ converges in the weak ${ }^{*}$ sense to $c$ in $X$, symbolically $c^{(n)} \dot{\Delta} c$, if (i) $\sup _{n}\left\|c^{(n)}\right\|<\infty$ and (ii) $c_{j}^{(n)} \rightarrow c_{j}$ as $n \rightarrow \infty$ for each $j$. The following characterization of weak* convergence is 
useful: let $y^{i} \in B_{\rho}$ be a sequence; then $y^{i} \dot{\Delta} y \in X$ as $i \rightarrow \infty$ if and only if $y \in B_{\rho}$ and $d\left(y^{i}, y\right) \rightarrow 0$ as $i \rightarrow \infty$.

Existence of solutions to (1.1) is proved by taking the limit as $n \rightarrow \infty$ of the truncated system

$$
\left\{\begin{aligned}
\dot{c}_{j}=\frac{1}{2} \sum_{k=1}^{j-1} W_{j-k . k}(c)-\sum_{k=1}^{n-j} W_{j, k}(c) & \\
c_{j}(0)=c_{0 j} \geqslant 0 & (1 \leqslant j \leqslant n)
\end{aligned}\right.
$$

A solution of (1.1) obtained as the limit of a solution of (2.1) is called an admissible solution.

\subsection{Preliminary Results}

We now state some basic results that will be needed afterward.

Proposition 2.1. (2) Assume (1.3.). Let $c_{0} \in X^{+}$. Then (1.1) has at least one admissible solution on $[0, \infty)$, and all admissible solutions conserve density. Furthermore, if for some $T>0, c$ is a density-conserving solution of $(1.1)$ on $[0, T)$, then $c \in \mathscr{C}^{0}([0, T), X)$ and $\sum_{j} j c_{j}(t)$ is uniformly convergent on compact sets of $[0, T)$.

Proposition 2.2. (2) Assume (1.3) and (1.7). Let $c_{0} \in X^{+}$, $T \in(0, \infty]$. Then, all solutions of $(1.1)$ on $[0, T)$ conserve density.

For the manipualtion of solutions the following results will be very useful.

Proposition 2.3 (Lemma 3.1 of ref. 2). Let $c$ be a solution of (1.1) on $[0, T)$ and $\left(g_{j}\right)$ be a sequence. Let $1 \leqslant m \leqslant n$ and $0 \leqslant t_{1}<t_{2}<T$. Then

$$
\begin{aligned}
\sum_{j=m}^{n} g_{j}\left(c_{j}\left(t_{2}\right)-c_{j}\left(t_{1}\right)\right)= & \int_{t_{1}}^{t_{2}}\left[\frac{1}{2} \sum_{R_{n, m}^{1}}\left(g_{j+k}-g_{j}-g_{k}\right)+\frac{1}{2} \sum_{R_{n, m}^{2}} g_{j+k}\right. \\
& \left.+\sum_{R_{n, m}^{3}}\left(g_{j+k}-g_{k}\right)-\sum_{R_{n, m}^{4}} g_{j}\right] W_{j, k}(c(s)) d s
\end{aligned}
$$

where

$$
\begin{aligned}
& R_{n, m}^{1}=\{(j, k): j, k \geqslant m, j+k \leqslant n\} \\
& R_{n, m}^{2}=\{(j, k): m \leqslant j+k \leqslant n, j, k<m\} \\
& R_{n, m}^{3}=\{(j, k): 1 \leqslant j \leqslant m-1, k \geqslant m, j+k \leqslant n\} \\
& R_{n, m}^{4}=\{(j, k): m \leqslant j \leqslant n, j+k>n\}
\end{aligned}
$$

with the sums equal to zero if the associated region is empty. 


\section{SOME PROPERTIES OF HIGHER MOMENTS}

Let $\mu \geqslant 1$ and define

$$
X_{\mu}=\left\{c \in X:\|c\|_{\mu} \stackrel{\text { def }}{=} \sum_{j=1}^{\infty} j^{\mu}\left|c_{j}\right|<\infty\right\}
$$

The spaces $\left(X_{\mu},\|\cdot\|_{\mu}\right)$ are Banach spaces and $X_{\mu} \subset X_{\nu}$ if $\mu>v$, with compact embedding. In this section we study the role of the hypotheses (1.3) and (1.7) on the regularity properties of solutions, as measured by the behavior of $\|c(t)\|_{\mu}$.

\subsection{Nonsmoothing of Higher Moments}

Theorem 3.1. Let $\mu>1,0<T \leqslant \infty$. Assume (1.7). Let $c$ be a density-conserving solution of $(1.1)$ on $[0, T)$ with initial data $c(0)=c_{0}$. If $c_{0} \notin X_{\mu}$, then $c(t) \notin X_{\mu}$ for all $t \in[0, T)$.

Proof. The proof will be based on estimates on

$$
T_{q}(t) \stackrel{\text { der }}{=} \sum_{n=1}^{q} \lambda_{n} S_{n}(t)
$$

where $\lambda_{n}=n^{v}-(n-1)^{v} \quad$ with $v=\mu-1>0$ and $S_{n}(t)=\sum_{r=n+1}^{\infty} r c_{r}(t)$. Writing $\lambda_{n} S_{n}=n^{\nu}\left(S_{n}-S_{n+1}\right)+n^{\nu} S_{n+1}-(n-1)^{v} S_{n}$, we find

$$
T_{q}=\sum_{n=2}^{q}(n-1)^{v} n c_{n}+q^{v} \sum_{n=q+1}^{\infty} n c_{n}
$$

Since the nature of the series $\sum_{j} j^{\mu} c_{j}$ and $\sum_{j}(j-1)^{\mu-1} j c_{j}$ are the same (both convergent or both divergent), to prove the theorem it is sufficient to show that

$$
\lim _{q \rightarrow \infty} T_{q}(0)=\infty \quad \text { implies } \quad \lim _{q \rightarrow \infty} T_{q}(t)=\infty
$$

for all $t>0$.

This will be done by estimating $T_{q}(t)$ using Gronwall's inequality. By density conservation we can write $S_{n}(t)=\rho_{0}-\sum_{r=1}^{n} r c_{r}(t)$, where $\rho_{0}$ is the density of the solution. Applying Proposition 2.3, we obtain

$$
\begin{aligned}
T_{q}(t) & =T_{q}(0)+\sum_{n=1}^{q} \lambda_{n}\left(S_{n}(t)-S_{n}(0)\right) \\
& =T_{q}(0)-\sum_{n=1}^{q} \lambda_{n} \sum_{r=1}^{n} r\left(c_{r}(t)-c_{r}(0)\right)
\end{aligned}
$$




$$
\begin{aligned}
& =T_{q}(0)+\int_{0}^{t} \sum_{n=1}^{q} \sum_{j=1}^{n} \sum_{k=n-j+1}^{\infty} j \lambda_{n} W_{j, k}(c(s)) d s \\
& \geqslant T_{q}(0)-\int_{0}^{l} \sum_{n=1}^{q} \sum_{j=1}^{n} \sum_{k=n-j+1}^{\infty} j \lambda_{n} b_{j, k} c_{j+k}(s) d s \\
& =T_{q}(0)-\int_{0}^{l} \sum_{n=1}^{q} \sum_{r=n+1}^{\infty} \lambda_{n} \gamma_{r, n} c_{r}(s) d s
\end{aligned}
$$

where $\gamma_{r, n}=\sum_{j=1}^{n} j b_{j, r-j}$. Changing the order of summation of the double sum in (3.3), we obtain

$$
\sum_{n=1}^{q} \sum_{r=n+1}^{\infty} \lambda_{n} \gamma_{r, n} c_{r}=\sum_{r=2}^{q} c_{r} \sum_{n=1}^{r-1} \lambda_{n} \gamma_{r, n}+\sum_{r=q+1}^{\infty} c_{r} \sum_{n=1}^{q} \lambda_{n} \gamma_{r, n}
$$

and in order to proceed we need to obtain estimates on the behavior of the coefficients of $c_{r}$ in (3.4). This is done in the next lemma, which we prove later.

Lemma 3.2. Assume (1.7). Then the following inequalities hold:

1. For $r \geqslant q+1, \sum_{n=1}^{q} \lambda_{n} \gamma_{r, n} \leqslant$ const $\cdot r q^{\nu}$.

2. For $2 \leqslant r \leqslant q, \sum_{n=1}^{r-1} \lambda_{n} \gamma_{r, n} \leqslant$ const $\cdot r(r-1)^{\nu}$.

Using the estimates of Lemma 3.2 in (3.4), substituting the result into (3.3), and using (3.2), we obtain

$$
-\left(T_{q}(t)-T_{q}(0)\right) \leqslant \mathscr{K} \int_{0}^{t} T_{q}(s) d s
$$

where $\mathscr{K}=\mathscr{K}\left(K_{f}, v\right)$ is a constant. Applying Gronwall's inequality, we obtain

$$
T_{q}(t) \geqslant T_{q}(0) e^{-\not{x}}
$$

and if $T_{q}(0) \rightarrow \infty$ as $q \rightarrow \infty$, then $T_{q}(t) \rightarrow \infty$ as $q \rightarrow \infty$ for all $t \in[0, T)$.

Proof of Lemma 3.2. Assume $r \geqslant 2 q-1$. Then $q \leqslant(r+1) / 2$, and since $q$ is an integer, this is the same as $q \leqslant h(r)$. We have

$$
\begin{aligned}
\sum_{n=1}^{q} \lambda_{n} \gamma_{r, n} & =\sum_{n=1}^{q} \lambda_{n} \sum_{j=1}^{n} j b_{j, r-j} \\
& \leqslant\left(\sum_{n=1}^{q} \lambda_{n}\right)\left(\sum_{j=1}^{h(r)} j b_{j, r-j}\right) \\
& =q^{\nu} \sum_{j=1}^{h(r)} j b_{j, r-j} \\
& \leqslant K_{f} q^{\nu} r
\end{aligned}
$$


Now let $q+1 \leqslant r \leqslant 2 q-2$.

Then $h(r)<(r+2) / 2 \leqslant q \leqslant r-1$, and we can write

$$
\begin{aligned}
\sum_{n=1}^{q} \lambda_{n} \gamma_{r, n} & =\sum_{n=1}^{q} \sum_{j=1}^{n} \lambda_{n} j b_{j, r-j} \\
& =\sum_{n=1}^{h(r)} \sum_{j=1}^{n} \lambda_{n} j b_{j, r-j}+\sum_{n=h(r)+1}^{q} \sum_{j=1}^{n} \lambda_{n} j b_{j, r-j}
\end{aligned}
$$

By changing the summation order we have, for the first double sum,

$$
\begin{aligned}
\sum_{n=1}^{h(r)} \sum_{j=1}^{n} \lambda_{n} j b_{j, r-j} & =\sum_{j=1}^{h(r)} b_{j, r-j} \sum_{n=j}^{h(r)} \lambda_{n} \\
& =h(r)^{v} \sum_{j=1}^{h(r)} j b_{j, r-j}-\sum_{j=1}^{h(r)} j(j-1)^{v} b_{j, r-j}
\end{aligned}
$$

and for the second double sum,

$$
\begin{aligned}
\sum_{n=h(r)+1}^{q} & \sum_{j=1}^{n} \lambda_{n} j b_{j, r-j} \\
= & \sum_{j=1}^{h(r)} \sum_{n=h(r)+1}^{q} \lambda_{n} j b_{j, r-j}+\sum_{j=h(r)+1}^{q} \sum_{n=j}^{q} \lambda_{n} j b_{j, r-j} \\
= & q^{v} \sum_{j=1}^{h(r)} j b_{j, r-j}-h(r)^{\nu} \sum_{j=1}^{h(r)} j b_{j, r-j} \\
& +q^{v} \sum_{j=h(r)+1}^{q} j b_{j, r-j}-\sum_{j=h(r)+1}^{q} j(j-1)^{v} b_{j, r-j}
\end{aligned}
$$

Substituting these expressions into (3.5), we obtain

$$
\begin{aligned}
\sum_{n=1}^{q} \lambda_{n} \gamma_{r, n} & =q^{\nu} \sum_{j=1}^{h(r)} j b_{j, r-j}+q^{\nu} \sum_{j=h(r)+1}^{q} j b_{j, r-j}-\sum_{j=1}^{q} j(j-1)^{\nu} b_{j, r-j} \\
& \leqslant K_{f} q^{\nu} r+\left[q^{\nu} \sum_{j=h(r)+1}^{q} j b_{j, r-j}-\sum_{j=1}^{q} j(j-1)^{\nu} b_{j, r-j}\right]
\end{aligned}
$$

For the terms in square brackets we have

$$
\begin{aligned}
q^{v} & \sum_{j=h(r)+1}^{q} j b_{j, r-j}-\sum_{j=1}^{q} j(j-1)^{v} b_{j, r-j} \\
& =\sum_{j=h(r)+1}^{q}\left(q^{v}-(j-1)^{v}\right) j b_{j, r-j}-\sum_{j=1}^{h(r)} j(j-1)^{v} b_{j, r-j}
\end{aligned}
$$




$$
\begin{aligned}
& \leqslant \sum_{j=h(r)+1}^{q}\left(q^{v}-(j-1)^{v}\right) j b_{j, r-j} \\
& \leqslant \sum_{j=h(r)+1}^{q}\left((r-1)^{v}-(j-1)^{v}\right) j b_{j, r-j}
\end{aligned}
$$

To estimate (3.7) we make use of the following inequalities:

$$
\begin{array}{ll}
(r-1)^{v}-(j-1)^{v} \leqslant v(r-1)^{v-1}(r-j), & v \geqslant 1 \\
(r-1)^{v}-(j-1)^{v} \leqslant v(j-1)^{v-1}(r-j), & 0<v<1
\end{array}
$$

For $v \geqslant 1,(3.7)$ becomes

$$
\begin{aligned}
& \sum_{j=h(r)+1}^{q}\left((r-1)^{\nu}-(j-1)^{\nu}\right) j b_{j, r-j} \\
& \leqslant v(r-1)^{v-1} \sum_{j=h(r)+1}^{q}(r-j) j b_{j, r-j} \\
& \leqslant v(r-1)^{v-1} \sum_{j=h(r)}^{q}(r-j) j b_{j, r-j} \\
& =v(r-1)^{v-1} \sum_{k=1}^{h(r)} k(r-k) b_{r-k, k} \\
& \leqslant v r^{\nu} \sum_{k=1}^{h(r)} k b_{r-k, k} \\
& \leqslant v 2^{v} q^{v} K_{f} r
\end{aligned}
$$

For $v \in(0,1)$, similar estimates show that

Hence,

$$
\sum_{j=h(r)+1}^{q}\left((r-1)^{v}-(j-1)^{v}\right) j b_{j, r-j} \leqslant v 2^{1-v} q^{v} K_{f} r
$$

$$
q^{v} \sum_{j=h(r)+1}^{q} j b_{j, r-j}-\sum_{j=1}^{q} j(j-1)^{v} b_{j, r-j} \leqslant \text { const } \cdot r
$$

and substituting this inequality into (3.6), we obtain

$$
\sum_{n=1}^{q} \lambda_{n} \gamma_{r, n} \leqslant \text { const } \cdot r
$$

which proves part 1 of the Lemma. Part 2 of the proof is similar, so we only indicate the main steps. Briefly, 


$$
\begin{aligned}
\sum_{n=1}^{r-1} \lambda_{n} \gamma_{r, n} & =\sum_{n=1}^{r-1} \sum_{j=1}^{n} \lambda_{n} j b_{j, r-j} \\
& =\sum_{j=1}^{r-1} \sum_{n=j}^{r-1} \lambda_{n} j b_{j, r-j} \\
& =\sum_{j=1}^{r-1}\left((r-1)^{\nu}-(j-1)^{\nu}\right) j b_{j, r-j}
\end{aligned}
$$

Writing the last sum as $\sum_{j=1}^{h(r)}+\sum_{j=h(r)+1}^{r-1}$, considering separately the cases $v \geqslant 1$ and $v \in(0,1)$, we finally obtain, for $r=2, \ldots, q$,

$$
\sum_{n=1}^{r-1} \lambda_{n} \gamma_{r, n} \leqslant \text { const } \cdot r
$$

\subsection{Absence of Blowup of Higher Moments of Admissible Solutions}

We now turn to the role of the hypothesis on the coagulation coefficients in the behavior of higher moments. We prove that, if a higher moment of an admissible solution is initially finite, it stays finite for all $t<\infty$. We need the following auxiliary results, a proof of which can be found in ref. 4: Let $\mu>1$; then there exists a constant $\mathscr{C}(\mu) \geqslant 0$ such that

$$
0 \leqslant(j+k)^{\mu}-j^{\mu}-k^{\mu} \leqslant \mathscr{C}(\mu)\left(j k^{\mu}+j^{\mu} k\right)(j+k)^{-1} \quad \text { for all } j \text { and } k \geqslant 1
$$

Theorem 3.3. Assume (1.3). Let $\mu>1$, and $c_{0} \in X^{+} \cap X_{\mu}$. Let $c$ be an admissible solution of $(1.1)$ on $[0, T)$ for some $T \in(0, \infty)$, with initial data $c(0)=c_{0}$. Then $c(t) \in X_{\mu}$ for all $t \in[0, T)$.

Proof. The proof will be based on estimates of the moments of the solution $c^{(n)}$ of the finite-dimensional system (2.1) with initial data $c^{(n)}(0)=$ $c_{0}^{(n)} \equiv\left(c_{01}, c_{02}, \ldots, c_{0 n}\right)$. By Lemma 2.1 of ref. 2 and Lemma 2.3 of ref. 4 and the above inequality,

$$
\begin{aligned}
\sum_{j=1}^{n} j^{\mu} \dot{c}_{j}^{(n)} & =\frac{1}{2} \sum_{j=1}^{n-1} \sum_{k=1}^{n-j}\left((j+k)^{\mu}-j^{\mu}-k^{\mu}\right) W_{j, k}\left(c^{(n)}\right) \\
& \leqslant \frac{1}{2} \sum_{j=1}^{n-1} \sum_{k=1}^{n-j}\left((j+k)^{\mu}-j^{\mu}-k^{\mu}\right) a_{j, k} c_{j}^{(n)} c_{k}^{(n)} \\
& \leqslant \mathscr{C}(\mu) K_{a} \sum_{j=1}^{n-1} \sum_{k=1}^{n-j} j^{\mu} k c_{j}^{(n)} c_{k}^{(n)} \\
& \leqslant \mathscr{C}(\mu) K_{a}\left\|c_{0}\right\| \sum_{j=1}^{n} j^{\mu} c_{j}^{(n)}
\end{aligned}
$$


where $\mathscr{C}(\mu)$ is a constant independent of $j$ and $k$. Hence, by Gronwall's inequality,

$$
\begin{aligned}
\sum_{j=1}^{n} j^{\mu} c_{j}^{(n)}(t) & \leqslant\left(\sum_{j=1}^{n} j^{\mu} c_{j}^{(n)}(0)\right) \exp \left(K_{a} \rho_{0} \mathscr{C}(\mu) t\right) \\
& \leqslant\left\|c_{0}\right\|_{\mu} \exp \left(K_{a} \rho_{0} \mathscr{C}(\mu) t\right)
\end{aligned}
$$

By admissibility of $c$ we can pass to the limit $n \rightarrow \infty$ in the previous inequality to obtain

$$
\sum_{j=1}^{\infty} j^{\mu} c_{j}(t) \leqslant\left\|c_{0}\right\|_{\mu} \exp \left(K_{a} \rho_{0} \mathscr{C}(\mu) t\right)
$$

\section{PURE FRAGMENTATION AND PURE COAGULATION: ASYMPTOTIC BEHAVIOR OF SOLUTIONS}

In this section we start the study of the behavior of solutions of (1.1) as $t \rightarrow \infty$. The cases of pure fragmentation and pure coagulation that we consider below are particularly easy to study due to the monotonicity of the function $t \mapsto \sum_{j=1}^{n} j c_{j}(t)$.

We start with the pure fragmentation case. It is heuristically clear that if each cluster can fragment in at least one way; then the final distribution consists only of monomers. This is proved in the next theorem.

Theorem 4.1. Let $a_{j, k}=0$ for all $j$ and $k$. Suppose that for each $j \geqslant 2$ there is a $k_{j}<j$ such that $b_{j-k_{j}, k_{j}}>0$. Suppose $c$ is the only solution of $(1.1)$ on $[0, \infty)$ with initial data $c_{0}$ and assume that $c$ conserves density on $[0, \infty)$. Define $\bar{c}=\left(\bar{c}_{j}\right)$ by $\bar{c}_{j}=\rho_{0} \delta_{j, 1}$, where $\rho_{0}=\left\|c_{0}\right\|$. Then

$$
c(t) \rightarrow \bar{c} \quad \text { strongly in } X \quad \text { as } t \rightarrow \infty
$$

Remark 4.2. By Theorems 3.6 and 4.2 of ref. 2 the assumption of existence, uniqueness, and density conservation of solutions to the pure fragmentation equations is satisfied under condition (1.7).

Proof of Theorem 4.1. Define

$$
p_{n}(t) \stackrel{\text { der }}{=} \sum_{j=1}^{n} j c_{j}(t)
$$

For all $n \geqslant 1$ and $t \geqslant 0, p_{n}(t) \leqslant \rho_{0}$. By Proposition 2.3 we have, for all $0 \leqslant t<\tau$,

$$
p_{n}(t+\tau)-p_{n}(t)=\int_{t}^{t+\tau} \sum_{j=1}^{n} \sum_{k=n-j+1}^{\infty} j b_{j, k} c_{j+k}(s) d s \geqslant 0
$$


and so $p_{n}(t)$ is nondecreasing in $t$ for each $n \geqslant 1$. Hence, there exist constants $\bar{p}_{n} \geqslant 0$ such that

$$
p_{n}(t) \rightarrow \bar{p}_{n} \quad \text { as } \quad t \rightarrow \infty
$$

and thus

$$
c_{n}(t) \rightarrow \bar{c}_{n} \quad \text { as } \quad t \rightarrow \infty
$$

with $\bar{c}_{1}=\bar{p}_{1}$ and $\bar{c}_{n}=\left(\bar{p}_{n}-\bar{p}_{n-1}\right) / n \geqslant 0$ for $n \geqslant 2$. By the sequentially weak* continuity of $c \mapsto \sum_{j=1}^{\infty} g_{j} c_{j}$, when $g_{j} \sim o(j)$ as $j \rightarrow \infty$, ${ }^{(3)}$ we conclude that

$$
\sum_{j=1}^{\infty} c_{j}(t) \rightarrow \sum_{j=1}^{\infty} \bar{c}_{j} \quad \text { as } \quad t \rightarrow \infty
$$

Hence, we can pass to the limit $t \rightarrow \infty$ in

$$
\sum_{j=1}^{\infty} c_{j}(t+1)-\sum_{j=1}^{\infty} c_{j}(t)=\frac{1}{2} \int_{t}^{t+1} \sum_{j=2}^{\infty} \sum_{k=1}^{j-1} b_{j-k, k} c_{j}(s) d s
$$

to obtain

$$
\begin{aligned}
0 & =\frac{1}{2} \lim _{t \rightarrow \infty} \int_{t}^{t+1} \sum_{j=2}^{\infty} \sum_{k=1}^{j-1} b_{j-k, k} c_{j}(s) d s \\
& \geqslant \frac{1}{2} \lim _{t \rightarrow \infty} \int_{t}^{t+1} \sum_{j=2}^{\infty} b_{j-k_{j}, k_{j}} c_{j}(s) d s \\
& \geqslant \frac{1}{2} b_{p-k_{p}, k_{p}} \lim _{t \rightarrow \infty} \int_{t}^{t+1} c_{p}(s) d s \quad \text { for all } p \geqslant 2
\end{aligned}
$$

Suppose there exists a $p \geqslant 2$ such that $\bar{c}_{p}>0$. Then, there exists $\alpha_{p}>0$ such that, for all $t$ sufficiently large, $c_{p}(t) \geqslant \alpha_{p}>0$, and thus

$$
0 \geqslant \frac{1}{2} b_{p-k_{p}, k_{p}} \lim _{t \rightarrow \infty} \int_{t}^{t+1} c_{p}(s) d s \geqslant \frac{1}{2} b_{p-k_{p}, k_{p}} \alpha_{p}>0
$$

Hence,

$$
\bar{c}_{j}=0 \quad \text { for all } j \geqslant 2
$$

For $\bar{c}_{1}$ observe that, by density conservation and the weak* lower semicontinuity of $\|\cdot\|$, we have

$$
\bar{c}_{1}=\|\bar{c}\| \leqslant \lim _{t \rightarrow \infty}\|c(t)\|=\rho_{0}
$$


Moreover,

$$
\bar{c}_{1}=\lim _{t \rightarrow \infty} p_{n}(t)=p_{n}(0)+\lim _{t \rightarrow \infty} \int_{0}^{t} \sum_{j=1}^{n} \sum_{k=n-j+1}^{\infty} j b_{j, k} c_{j+k}(s) d s \geqslant p_{n}(0)
$$

and thus

$$
\bar{c}_{1} \geqslant \lim _{n \rightarrow \infty} p_{n}(0)=\rho_{0}
$$

Hence, (4.2)-(4.4) imply $\bar{c}_{j}=\rho_{0} \delta_{j .1}$, and the strong convergence follows by Lemma 3.3 of ref. 3.

We now consider the pure coagulation case.

Theorem 4.3. Let $b_{j . k}=0$ for all $j$ and $k$, and assume $a_{j . j}>0$ for all $j \geqslant 1$. Let $c$ be a solution of $(1.1)$ on $[0, \infty)$ with $c(0)=c_{0} \in X^{+}$. Then $c(t) \dot{\rightarrow} 0$ as $t \rightarrow \infty$.

Proof. With $p_{n}$ as above, by Proposition 2.3,

$$
p_{n}(t+\tau)-p_{n}(t)=-\int_{t}^{t+\tau} \sum_{j=1}^{n} \sum_{k=n-j+1}^{\infty} j a_{j, k} c_{j}(s) c_{k}(s) d s \leqslant 0
$$

so $p_{n}(t)$ is a nonincreasing function of $t$ for all $t \geqslant 0$ and all $n \geqslant 1$, and since $p_{n}(t) \geqslant 0$ there exist constants $\bar{p}_{n} \geqslant 0$ such that

$$
p_{n}(t) \rightarrow \bar{p}_{n} \quad \text { as } \quad t \rightarrow \infty
$$

for all $n \geqslant 1$. Hence $c_{n}(t) \rightarrow \bar{c}_{n}$ as $t \rightarrow \infty$ for all $n \geqslant 1$, with $\bar{c}_{n}$ as in the proof of Theorem 4.1. We prove that $\bar{c}_{n}=0$ for all $n$ by induction:

If $n=1$, since $c_{1}(t)=p_{1}(t)$, we conclude, by (4.5), that $c_{1}$ is nonincreasing, thus $c_{1}(t) \geqslant \bar{c}_{1} \geqslant 0$. For all $t \geqslant 0$ and $\tau>0$,

$$
\begin{aligned}
c_{1}(t+\tau)-c_{1}(t) & =-\int_{t}^{t+\tau} \sum_{k=1}^{\infty} a_{1, k} c_{1}(s) c_{k}(s) d s \\
& =-\int_{t}^{t+\tau} \sum_{k=2}^{\infty} a_{1, k} c_{1}(s) c_{k}(s) d s-a_{1,1} \int_{t}^{t+\tau}\left(c_{1}(s)\right)^{2} d s \\
& \leqslant-a_{1,1} \int_{t}^{t+\tau}\left(c_{1}(s)\right)^{2} d s \\
& \leqslant-a_{1,1} \bar{c}_{1}^{2} \tau \leqslant 0
\end{aligned}
$$

Letting $t \rightarrow \infty$, we obtain $0 \leqslant a_{1,1} \bar{c}_{1}^{2} \tau \leqslant 0$, implying $\bar{c}_{1}=0$. 
For $n=2$,

$$
\begin{aligned}
c_{2}(t+\tau)-c_{2}(t)= & \int_{t}^{t+\tau}\left(\frac{1}{2} a_{1,1}\left(c_{1}(s)\right)^{2}-c_{2}(s) \sum_{k=1}^{\infty} a_{2, k} c_{k}\right) d s \\
= & \int_{1}^{t+\tau} c_{1}(s)\left(\frac{1}{2} a_{1,1} c_{1}(s)-a_{2,1} c_{2}(s)\right) d s \\
& -\int_{t}^{t+\tau} c_{2}(s) \sum_{k=3}^{\infty} a_{2, k} c_{k} d s \\
& -a_{2,2} \int_{t}^{t+\tau}\left(c_{2}(s)\right)^{2} d s \\
\leqslant & \int_{t}^{t+\tau} c_{1}(s)\left(\frac{1}{2} a_{1,1} c_{1}(s)-a_{2,1} c_{2}(s)\right) d s \\
& -a_{2,2} \int_{1}^{t+\tau}\left(c_{2}(s)\right)^{2} d s
\end{aligned}
$$

Letting $t \rightarrow \infty$, we have

$$
\begin{aligned}
0 \leqslant & \lim _{t \rightarrow \infty} \int_{t}^{t+\tau} c_{1}(s)\left(\frac{1}{2} a_{1,1} c_{1}(s)-a_{2,1} c_{2}(s)\right) d s \\
& -a_{2,2} \lim _{t \rightarrow \infty} \int_{t}^{t+\tau}\left(c_{2}(s)\right)^{2} d s
\end{aligned}
$$

and since $c_{1} \rightarrow 0$ as $t \rightarrow \infty$ and $c_{2}(t)$ is bounded, the right-hand side of (4.6) is zero and we have

$$
0 \leqslant-a_{2,2} \lim _{t \rightarrow \infty} \int_{t}^{t++}\left(c_{2}(s)\right)^{2} d s
$$

Hence

$$
\lim _{t \rightarrow \infty} \int_{1}^{1+\tau}\left(c_{2}(s)\right)^{2} d s=0
$$

and this implies $\vec{c}_{2}=0$, because otherwise there would exist a positive constant $0<\alpha_{2}<\bar{c}_{2}$ such that, for all sufficiently large $t, c_{2}(t)>\alpha_{2}$ and

$$
\lim _{t \rightarrow \infty} \int_{t}^{t+\tau}\left(c_{2}(s)\right)^{2} d s>\left(\alpha_{2}\right)^{2} \tau>0
$$

a contradiction. 
Proceeding by induction, assuming $\bar{c}_{1}=\cdots=\bar{c}_{n-1}=0$, we prove $\bar{c}_{n}=0$ :

$$
\begin{aligned}
0 & =\lim _{t \rightarrow \infty}\left(c_{n}(t+\tau)-c_{n}(t)\right) \\
& =\lim _{t \rightarrow \infty}\left\{\int_{t}^{t+\tau}\left[\frac{1}{2} \sum_{k=1}^{n-1} a_{n-k, k} c_{n-k}(s) c_{k}(s)-c_{n}(s) \sum_{k=1}^{\infty} a_{n, k} c_{k}(s)\right] d s\right\} \\
& \leqslant-a_{n, n} \lim _{t \rightarrow \infty} \int_{t}^{t+\tau}\left(c_{n}(s)\right)^{2} d s
\end{aligned}
$$

and the conclusion follows as before.

\section{COAGULATION-FRAGMENTATION: EQUILIBRIA AND LYAPUNOV FUNCTIONS}

By an equilibrium we mean a time-independent solution of (1.1). If we assume the detailed balance condition (1.6), then for any $c_{1} \geqslant 0$ the sequence $c=\left(c_{j}\right)$ defined by

$$
c_{j}=Q_{j}\left(c_{1}\right)^{j}, \quad j \geqslant 2
$$

satisfies $a_{j, k} c_{j} c_{k}-b_{j, k} c_{j+k}=0$ for all $j, k$ and thus $c$ is an equilibrium of (1.1) provided $c \in X$, i.e., $c_{1}$ must be chosen in such a way that

$$
\|c\|=\sum_{j=1}^{\infty} j c_{j}=\sum_{j=1}^{\infty} j Q_{j} c_{1}^{j}<\infty
$$

Let $z \geqslant 0$ and set

$$
F(z)=\sum_{j=1}^{\infty} j Q_{j} z^{j}
$$

Let $\rho_{s}=\sup _{0 \leqslant z<z_{s}} F(z)$, where

$$
z_{s}=\left(\limsup _{j \rightarrow \infty} Q_{j}^{1 / j}\right)^{-1}
$$

is the radius of convergence of the series in the right-hand side of (5.2). If $z_{s}=\infty$, then $\rho_{s}=\infty$. In the case $z_{s} \in(0, \infty)$ we have $\rho_{s} \in(0, \infty]$ and if $\rho_{s}<\infty$ then $\rho_{s}=F\left(z_{s}\right)$. For $c \in X^{+}$let

$$
V(c)=\sum_{j=1}^{\infty} c_{j}\left(\log \frac{c_{j}}{Q_{j}}-1\right)
$$


where the summand is defined to be zero when $c_{j}=0$. Define

$$
V_{z}(c)=V(c)-\left(\sum_{j=1}^{\infty} j c_{j}\right) \log z
$$

In order to show that $V$ is a Lyapunov function, we need to introduce some more hypotheses on the rate coefficients:

H1. $a_{j, k} \leqslant K_{c}\left(j^{\alpha}+k^{\alpha}\right)$ for all $j, k \geqslant 1$, where $K_{c}>0$ and $0 \leqslant \alpha<1$ are constants.

H2. $\sum_{j=1}^{h(r)} b_{j, r-j} \leqslant \kappa_{f} r^{\beta}$ for all $r \geqslant 2$, where $\kappa_{f} \geqslant 0$ and $\beta \in(0,1)$ are constants.

H3. For all $k \geqslant 1, a_{1, k}, b_{1, k}>0$.

H4. The sequence $Q_{j}$ defined in (1.6) satisfies

1. $\left|\log Q_{j+1}-\log Q_{j}\right| \leqslant K_{Q}$ for some constant $K_{Q} \geqslant 0$ and all $j \geqslant 1$.

2. $\log Q_{j}+\log Q_{k} \leqslant \log Q_{j+k}$ for all $j, k \geqslant 1$.

3. $0<\lim \inf _{j \rightarrow \infty} Q_{j}^{1 / j} \leqslant \lim \sup _{j \rightarrow \infty} Q_{j}^{1 / j}<\infty$.

Remark 5.1. Assumption $\mathrm{H} 3$ implies all solutions are strictly positive for $t>0$, i.e., $c_{j}(t)>0$ for all $j \geqslant 1$ and $t>0 .{ }^{(4)}$ Conditions H4.1 and $\mathrm{H} 4.2$ have the following physical interpretation. Since the quantity $-\log Q_{j}$ is the free energy of a cluster size $j$, condition $\mathrm{H} 4.1$ means that the energy change involved in the gain of a single particle by a $j$-cluster is bounded independent of $j$. Condition $\mathrm{H} 4.2$ is a statement on the thermodynamic stability of clusters: a given cluster has a lower free energy than the sum of the free energies of all the possible clusters that can be obtained via fragmentation.

Theorem 5.2. Assume (1.7) and $\mathrm{H} 1-\mathrm{H} 4$. Let $c$ be a solution of (1.1) on $[0, T)$ with initial data $c_{0} \neq 0$. Then

$$
V(c(t))=V(c(0))-\int_{0}^{t} D(c(s)) d s
$$

where

$$
D(c)=\sum_{j, k=1}^{\infty} W_{j . k}(c)\left[\log \left(Q_{j+k} c_{j} c_{k}\right)-\log \left(Q_{j} Q_{k} c_{j+k}\right)\right] \geqslant 0
$$

Proof. The basic idea of the proof is similar to the corresponding ones in refs. 3 and 4. Defining

$$
V^{n}(c)=\sum_{j=1}^{n} c_{j}\left(\log \frac{c_{j}}{Q_{j}}-1\right)
$$


we obtain an equation for the evolution of $V^{n}$ along solutions of (1.1). From this equation we deduce two inequalities for the asymptotic behavior of $V^{n}(c)$ when $n \rightarrow \infty$. Using these inequalities and the monotone convergence theorem, we prove (5.5).

We start by proving the inequality in (5.6). If $a_{j . k} \neq 0$, then by the detailed balance condition (1.6), $b_{j, k} \neq 0$. Assumption $\mathrm{H} 3$ implies that all solutions satisfy $c_{j}(t)>0$ for all $t>0, j \geqslant 1 .^{(4)}$ Since $(x-y)(\log x-\log y)$ $>0$ for all $x, y>0, x \neq y$, we conclude that

$$
\begin{aligned}
& W_{j, k}(c)\left[\log \left(Q_{j+k} c_{j} c_{k}\right)-\log \left(Q_{j} Q_{k} c_{j+k}\right)\right] \\
& \quad=W_{j, k}(c)\left[\log \left(a_{j, k} c_{j} c_{k}\right)-\log \left(b_{j, k} c_{j+k}\right)\right] \geqslant 0
\end{aligned}
$$

If for some $\tilde{j}$ and $\tilde{k}, a_{j, \bar{k}}=0$, then (1.6) implies $b_{j, \bar{k}}=0$, too, so that $W_{j, \tilde{k}}(c)=0$ and the corresponding term in the sum (5.6) is zero. This proves the inequality.

Define

$$
D_{n}(c)=\frac{1}{2} \sum_{j+k \leqslant n} W_{j, k}(c)\left[\log \left(Q_{j+k} c_{j} c_{k}\right)-\log \left(Q_{j} Q_{k} c_{j+k}\right)\right]
$$

Condition $\mathrm{H} 2$ implies that $b_{j, k} \leqslant$ const $\cdot j k$. In particular this implies, by Theorem 5.2 of ref. 2, that for density-conserving solutions each component $c_{j}$ is continuously differentiable. Thus, by Proposition 2.3 with $g_{j}=\log \left(c_{j} / Q_{j}\right)$ we obtain, for all $0<\tau \leqslant t<T$,

$$
\begin{aligned}
& V^{n}(c(t))-V^{n}(c(\tau)) \\
& \quad=-\int_{\tau}^{t} D_{n}(c(s)) d s-\int_{\tau}^{t} \sum_{j=1}^{n} \sum_{k=n-j+1}^{\infty}\left(\log \frac{c_{j}(s)}{Q_{j}}\right) W_{j . k}(c(s)) d s
\end{aligned}
$$

We use (5.9) to obtain the following inequalities that will allow us to pass to the limit $n \rightarrow \infty$ :

$$
\begin{array}{ll}
V^{n}(c(t)) \leqslant V^{n}(c(\tau))-\int_{\tau}^{t} D_{n}(c(s)) d s+o(1) & \text { as } \quad n \rightarrow \infty \\
V^{n}(c(t)) \geqslant V^{n}(c(\tau))-\int_{\tau}^{t} D_{n}(c(s)) d s+o(1) & \text { as } \quad n \rightarrow \infty
\end{array}
$$

valid for all $\tau, t$ satisfying $0<\tau \leqslant t<T$.

We start by proving (5.10). The double sum in the right-hand side of (5.9) can be written as

$$
\sum_{j=1}^{n} \sum_{k=n-j+1}^{\infty} W_{j, k}(c) \log c_{j}-\sum_{j=1}^{n} \sum_{k=n-j+1}^{\infty} W_{j, k}(c) \log Q_{j}
$$


By H4.1 and Theorem 3.7 of ref. 2,

$$
\int_{\tau}^{1} \sum_{j=1}^{n} \sum_{k=n-j+1}^{\infty} W_{j, k}(c(s)) \log Q_{j} d s \rightarrow 0 \quad \text { as } n \rightarrow \infty
$$

For the first double sum in (5.12) we can write

$$
\begin{aligned}
\int_{\tau}^{\ell} \sum_{j=1}^{n} & \sum_{k=n-j+1}^{\infty} W_{j, k}(c(s)) \log c_{j}(s) d s \\
= & \int_{\tau}^{l} \sum_{j=1}^{\prime n 0-1} \sum_{k=n-j+1}^{\infty} W_{j, k}(c(s)) \log c_{j}(s) d s \\
& +\int_{\tau}^{l} \sum_{j=n}^{n} \sum_{k=n-j+1}^{\infty} W_{j, k}(c(s)) \log c_{j}(s) d s
\end{aligned}
$$

for some fixed $n_{0}>1$.

Observe that, for all $\varepsilon>0$, there exists a constant $\mathscr{C}(\varepsilon)>0$ such that

$$
x|\log x| \leqslant \mathscr{C}(\varepsilon)\left(x^{1+\varepsilon}+x^{1-\varepsilon}\right)
$$

for all $x>0$.

Since $c_{j}(s)$ is bounded and bounded away from zero for $s$ in compact intervals not containing the origin, we conclude that $\left|\log c_{j}(s)\right|$ is bounded for $1 \leqslant j \leqslant n_{0}-1$ and $\tau \leqslant s \leqslant t$. Hence, by definition of solution, the integral (5.15) converges to zero as $n \rightarrow \infty$. Thus, in order to prove (5.10), we need to prove that

$$
-\int_{\tau}^{l} \sum_{j=n_{0}}^{n} \sum_{k=n-j+1}^{\infty} W_{j, k}(c(s)) \log c_{j}(s) d s \leqslant o(1) \quad \text { as } n \rightarrow \infty
$$

The series $\sum_{j} j c_{j}(t)$ is uniformly convergent on compact subsets of $[0, T)$, and so, given $\mathscr{I}$ a compact subset of $[0, T)$, there exists an integer $n_{0}$ such that, for all $s \in \mathscr{I}$ and all $j \geqslant n_{0}$

$$
-\log c_{j}(s)>0
$$

So, choosing $n_{0}$ in (5.18) conveniently, we have

$$
\begin{aligned}
-\log c_{j}(s) W_{j, k}(c(s)) & =-\log c_{j}(s)\left(a_{j, k} c_{j}(s) c_{k}(s)-b_{j, k} c_{j+k}(s)\right) \\
& \leqslant a_{j, k} c_{k}(s) c_{j}(s)\left|\log c_{j}(s)\right|
\end{aligned}
$$

Considering that, for sufficiently large $j$ and all $s, c_{j}(s)<1$, we have, by (5.17),

$$
-\log c_{j}(s) W_{j, k}(c(s)) \leqslant 2 \mathscr{C}(\varepsilon) a_{j, k} c_{k}(s) c_{j}(s)^{1-\varepsilon}
$$


Hence, using $\mathrm{Hl}$ and (5.21),

$$
\begin{aligned}
\int_{\tau}^{t} \sum_{j=n_{0}}^{n} \sum_{k=n-j+1}^{\infty} W_{j, k}(c(s)) \log c_{j}(s) d s \\
\leqslant 2 \mathscr{C}(\varepsilon) K_{c} \int_{\tau}^{t} \sum_{j=n_{0}}^{n} \sum_{k=n-j+1}^{\infty}\left(j^{\alpha}+k^{\alpha}\right) c_{k}(s) c_{j}(s)^{1-\varepsilon} d s \\
=2 \mathscr{C}(\varepsilon) K_{c} \int_{\tau}^{t}\left[\sum_{j=n_{0}}^{n} j^{\alpha} c_{j}(s)^{1-\varepsilon} \sum_{k=n-j+1}^{\infty} c_{k}(s)\right. \\
\left.\quad+\sum_{j=n_{0}}^{n} c_{j}(s)^{1-\varepsilon} \sum_{k=n-j+1}^{\infty} k^{\alpha} c_{k}(s)\right] d s
\end{aligned}
$$

By Hölder's inequality

$$
\sum_{j=1}^{n} j^{\sigma} c_{j}^{1-\varepsilon} \leqslant\left(\sum_{j=1}^{n} j c_{j}\right)^{1-\varepsilon} \cdot\left(\sum_{j=1}^{n} j^{1+(\sigma-1) / \varepsilon}\right)^{\varepsilon}
$$

for all $\sigma \in[0,1)$ and $\varepsilon \in(0,1)$. The first term in the right-hand side of (5.25) is bounded by $\left\|c_{0}\right\|^{1-\varepsilon}$, and if $\varepsilon \in(0,(1-\sigma) / 2)$, the second sum is convergent as $n \rightarrow \infty$. Hence, we can bound (5.25) by a constant $\mathscr{C}=\mathscr{C}\left(\left\|c_{0}\right\|, \varepsilon, \sigma\right)$ independent of $s$. Taking $\sigma=\alpha$, we conclude that $\sum_{j, k} j^{\alpha} c_{j}^{1-\varepsilon} c_{k}$ is uniformly convergent and so the remainders in the righthand side of (5.24) converge to zero as $n \rightarrow \infty$. This proves (5.18), and hence (5.10).

Knowing that $(5.10)$ holds, we can prove that

$$
\int_{\tau}^{l} D_{n}(c(s)) d s \rightarrow \int_{\tau}^{t} D(c(s)) d s \quad \text { as } \quad n \rightarrow \infty
$$

with $D$ and $D_{n}$ given by (5.6) and (5.8), respectively. By Lemma 4.2 of ref. 3 and the present assumptions on the asymptotic of $Q_{j}^{1 / j}$, we conclude that $V(c(\cdot))$ is bounded, and by $(5.10)$,

$$
\int_{\tau}^{t} D_{n}(c(s)) d s \leqslant V^{n}(c(\tau))-V^{n}(c(t))+o(1) \quad \text { as } \quad n \rightarrow \infty
$$

Since $V^{n}(c(s)) \rightarrow V(c(s))$ as $n \rightarrow \infty$, we can bound the right-hand side of (5.27) independently of $n$. Also, for all $s$ and $n, D_{n-1}(c(s)) \leqslant D_{n}(c(s)) \leqslant$ $D(c(s))$, and $D_{n}(c(s)) \rightarrow D(c(s))$ as $n \rightarrow \infty$, so we can apply the monotone convergence theorem and conclude that $D(c(\cdot))$ is integrable in $[\tau, t]$ and (5.26) holds.

In order to prove (5.11) we make use of the integrability of $D(c(\cdot))$. We need to prove that 


$$
-\int_{\tau}^{t} \sum_{j=1}^{n} \sum_{k=n-j+1}^{\infty} \log \frac{c_{j}(s)}{Q_{j}} W_{j, k}(c(s)) d s \geqslant o(1) \quad \text { as } n \rightarrow \infty
$$

Since $\int_{\tau}^{t} D(c(s)) d s<\infty$ we have

$$
\begin{aligned}
\lim _{n \rightarrow \infty} & \int_{\tau}^{t} \sum_{j=1}^{n} \sum_{k=n-j+1}^{\infty} W_{j, k}(c(s)) \\
& \times\left[\log \left(Q_{j+k} c_{j}(s) c_{k}(s)\right)-\log \left(Q_{j} Q_{k} c_{j+k}(s)\right)\right] d s=0
\end{aligned}
$$

Hence, as $n \rightarrow \infty$,

$$
\begin{aligned}
-\int_{\tau} \sum_{j=1}^{n} \sum_{k=n-j+1}^{\infty} \log \frac{c_{j}(s)}{Q_{j}} W_{j, k}(c(s)) d s & \\
= & \int_{\tau}^{t} \sum_{j=1}^{n} \sum_{k=n-j+1}^{\infty} W_{j, k}(c(s))\left[\log \frac{Q_{j+k} c_{j}(s) c_{k}(s)}{Q_{j} Q_{k} c_{j+k}(s)}\right. \\
& \left.\quad-\log \frac{c_{j}(s)}{Q_{j}(s)}\right] d s+o(1) \\
= & \int_{\tau}^{t} \sum_{j=1}^{n} \sum_{k=n-j+1}^{\infty} W_{j, k}(c(s)) \log \frac{Q_{j+k} c_{k}(s)}{Q_{k} c_{j+k}(s)} d s+o(1) \\
= & -\int_{\tau}^{t} \sum_{j=1}^{n} \sum_{k=n-j+1}^{\infty} W_{j, k}(c(s)) \log c_{j+k}(s) d s \\
& +\int_{\tau}^{t} \sum_{j=1}^{n} \sum_{k=n-j+1}^{\infty} W_{j, k}(c(s)) \log c_{k}(s) d s \\
& +\int_{\tau}^{t} \sum_{j=1}^{n} \sum_{k=n-j+1}^{\infty} W_{j, k}(c(s)) \log \frac{Q_{j+k}}{Q_{k}} d s+o(1)
\end{aligned}
$$

We are going to deal with each of (5.29)-(5.31) separately. Starting with (5.29), we have, from (5.19) and the paragraph that precedes it, that by choosing $n_{0}$ sufficiently large

$$
\begin{aligned}
& -\int_{\tau}^{1} \sum_{j=1}^{n} \sum_{k=n-j+1}^{\infty} W_{j, k}(c(s)) \log c_{j+k}(s) d s \\
& \quad=-\int_{\tau}^{l} \sum_{j=1}^{n} \sum_{k=n-j+1}^{\infty}\left(a_{j, k} c_{j}(s) c_{k}(s)-b_{j, k} c_{j+k}(s)\right) \log c_{j+k}(s) d s \\
& \quad \geqslant \int_{\tau}^{l} \sum_{j=1}^{n} \sum_{k=n-j+1}^{\infty} b_{j, k} c_{j+k}(s) \log c_{j+k}(s) d s \\
& \quad=-\int_{\tau}^{l} \sum_{j=1}^{n} \sum_{k=n-j+1}^{\infty} b_{j, k} c_{j+k}(s)\left|\log c_{j+k}(s)\right| d s \\
& \geqslant-2 \mathscr{C}(\varepsilon) \int_{\tau}^{\prime} \sum_{j=1}^{n} \sum_{k=n-j+1}^{\infty} b_{j, k} c_{j+k}^{1-\varepsilon}(s) d s
\end{aligned}
$$


using (5.17) for the last inequality. In order to prove that (5.32) converges to zero as $n \rightarrow \infty$, it is sufficient to prove the uniform convergence of the series $\sum_{j, k} b_{j, k} c_{j+k}^{1-\varepsilon}$. Using $\mathrm{H} 2$ and noticing that $r-h(r)-1<h(r)$ and $h(r)+1 \leqslant r-1$ for $r \geqslant 4$, we have

$$
\begin{aligned}
\sum_{j+k \leqslant n} & b_{j, k} c_{j+k}^{1-\varepsilon} \\
& =\sum_{r=2}^{n} c_{r}^{1-\varepsilon} \sum_{j=1}^{r-1} b_{j, r-j} \\
& =\sum_{r=2}^{3} c_{r}^{1-\varepsilon} \sum_{j=1}^{r-1} b_{j, r-j}+\sum_{r=4}^{n} c_{r}^{1-\varepsilon} \sum_{j=1}^{r-1} b_{j, r-j} \\
& =\sum_{r=2}^{3} c_{r}^{1-\varepsilon} \sum_{j=1}^{r-1} b_{j, r-j}+\sum_{r=4}^{n} c_{r}^{1-\varepsilon}\left(\sum_{j=1}^{h(r)} b_{j, r-j}+\sum_{j=h(r)+1}^{r-1} b_{j, r-j}\right) \\
& =\sum_{r=2}^{3} c_{r}^{1-\varepsilon} \sum_{j=1}^{r-1} b_{j, r-j}+\sum_{r=4}^{n} c_{r}^{1-\varepsilon}\left(\sum_{j=1}^{h(r)} b_{j, r-j}+\sum_{l=1}^{r-h(r)-1} b_{r-1,1}\right) \\
& \leqslant \sum_{r=2}^{3} c_{r}^{1-\varepsilon} \sum_{j=1}^{r-1} b_{j, r-j}+2 \kappa_{f} \sum_{r=4}^{n} r^{\beta} c_{r}^{1-\varepsilon}
\end{aligned}
$$

and by (5.25) with $\sigma=\beta$ and $\varepsilon \in(0,(1-\beta) / 2)$ we conclude that

$$
\sum_{j=1}^{n} j^{\beta} c_{j}^{1-\varepsilon}(s) \leqslant \mathscr{C}<\infty
$$

where $\mathscr{C}=\mathscr{C}\left(\left\|c_{0}\right\|, \varepsilon, \beta\right)$ is independent of $n$ and $s$, for $s$ in compact subsets of $(0, T)$, which proves the right-hand side of $(5.32)$ is $o(1)$ as $n \rightarrow \infty$.

For (5.30) we have

$$
\begin{aligned}
\int_{\tau}^{t} \sum_{j=1}^{n} & \sum_{k=n-j+1}^{\infty} W_{j, k}(c(s)) \log c_{k}(s) d s \\
= & \int_{\tau}^{t} \sum_{k=1}^{n_{0}-1} \sum_{j=n-k+1}^{n} W_{j, k}(c(s)) \log c_{k}(s) d s \\
& \quad+\int_{\tau}^{t} \sum_{j=1}^{n} \sum_{\substack{k=n-j+1 \\
k \geqslant n_{0}}}^{\infty} W_{j, k}(c(s)) \log c_{k}(s) d s
\end{aligned}
$$

The integral (5.34) converges to zero as $n \rightarrow \infty$ for the same reason that (5.15) does. For (5.35) observe that 


$$
\begin{aligned}
\sum_{j=1}^{n} & \sum_{\substack{k=n-j+1 \\
k \geqslant n_{0}}}^{\infty} W_{j, k}(c(s)) \log c_{k}(s) d s \\
& \geqslant-\sum_{j=1}^{n} \sum_{\substack{k=n-j+1 \\
k \geqslant n_{0}}}^{\infty} a_{j, k} c_{j}(s) c_{k}(s)\left|\log c_{k}(s)\right| d s
\end{aligned}
$$

and using (5.17), $\mathrm{H1}$, and the argument that follows (5.24) we conclude the right-hand side of (5.36) tends to zero as $n \rightarrow \infty$.

Finally, for (5.31) observe first that, by H4.1 and Theorem 3.7 of ref. 2, we know that

$$
\lim _{n \rightarrow \infty} \int_{\tau}^{t} \sum_{j=1}^{n} \sum_{k=n-j+1}^{\infty} W_{j, k}(c(s)) \log Q_{j}(s) d s=0
$$

So, to prove (5.31), it suffices to show that

$$
\begin{gathered}
\int_{\tau}^{1} \sum_{j=1}^{n} \sum_{k=n-j+1}^{\infty} W_{j, k}(c(s))\left[\log Q_{j+k}-\log Q_{j}-\log Q_{k}\right] d s \\
\geqslant o(1) \quad \text { as } n \rightarrow \infty
\end{gathered}
$$

Let

$$
\alpha_{j, k} \stackrel{\text { der }}{=} \log Q_{j+k}-\log Q_{j}-\log Q_{k}
$$

By H4.2 $\alpha_{j, k} \geqslant 0$, and since $W_{j, k}(c(s)) \geqslant-b_{j, k} c_{j+k}(s)$, we conclude

$$
W_{j, k}(c(s)) \alpha_{j, k} \geqslant-\alpha_{j, k} b_{j, k} c_{j+k}(s)
$$

Now consider the series $\sum_{j, k} \alpha_{j, k} b_{j, k} c_{j+k}$. A partial sum of this series can be written as follows:

$$
\begin{aligned}
\sum_{j+k \leqslant n} \alpha_{j, k} b_{j, k} c_{j+k}= & \sum_{r=2}^{n} c_{r} \sum_{j=1}^{r-1} \alpha_{j, r-j} b_{j, r-j} \\
= & \sum_{r=2}^{3} c_{r} \sum_{j=1}^{r-1} \alpha_{j, r-j} b_{j, r-j}+\sum_{r=4}^{n} c_{r} \sum_{j=1}^{r-1} \alpha_{j, r-j} b_{j, r-j} \\
= & \sum_{r=2}^{3} c_{r} \sum_{j=1}^{r-1} \alpha_{j, r-j} b_{j, r-j}+\sum_{r=4}^{n} c_{r} \sum_{j=1}^{h(r)} \alpha_{j, r-j} b_{j, r-j} \\
& +\sum_{r=4}^{n} c_{r} \sum_{j=h(r)+1}^{r-1} \alpha_{j, r-j} b_{j, r-j} \\
\leqslant & \sum_{r=2}^{3} c_{r} \sum_{j=1}^{r-1} \alpha_{j, r-j} b_{j, r-j}+2 \sum_{r=4}^{n} c_{r} \sum_{j=1}^{h(r)} \alpha_{j, r-j} b_{j, r-j}
\end{aligned}
$$


By $\mathrm{H} 4.1$ and $\mathrm{H} 4.2$ we have

$$
\begin{aligned}
\alpha_{j, r-j} & =\left|\log Q_{r-j}+\log Q_{j}-\log Q_{r}\right| \\
& \leqslant\left|\log Q_{r-j}-\log Q_{r}\right|+\left|\log Q_{j}\right| \\
& \leqslant K_{Q} j+\left|\log Q_{j}\right|
\end{aligned}
$$

By H4.3 there exist constants $\kappa_{\text {inf }}, \kappa_{\text {sup }}$ such that, for all $j$,

$$
e^{\kappa_{\mathrm{in} f}} \leqslant Q_{j}^{1 / j} \leqslant e^{\kappa_{\text {sup }}}
$$

It follows that

$$
\left|\log Q_{j}\right| \leqslant \kappa j
$$

where $\kappa=\max \left\{\left|\kappa_{\text {inf }}\right|,\left|\kappa_{\text {sup }}\right|\right\}$. Thus, there exists a $K_{\alpha} \geqslant K_{Q}+\kappa$ such that $\alpha_{j, r-j} \leqslant K_{\alpha} j$ for all $j$. Substituting this estimate into (5.39) and using (1.7), we get

$$
\sum_{r=4}^{n} c_{r} \sum_{j=1}^{h(r)} \alpha_{j, r-j} b_{j, r-j} \leqslant K_{\alpha} K_{f} \sum_{r=4}^{n} r c_{r}
$$

and hence the uniform convergence of the series. This proves (5.37), and concludes the proof of (5.11).

From (5.10) and (5.11) we have, for $0<\tau \leqslant t<T$,

$$
-\int_{\tau}^{t} D_{n}(c(s)) d s+o(1) \leqslant V^{n}(c(t))-V^{n}(c(\tau)) \leqslant-\int_{\tau}^{t} D_{n}(c(s)) d s+o(1)
$$

as $n \rightarrow \infty$. By the convergence of $V^{n}$ to $V$ and (5.26) we can pass to the limit $n \rightarrow \infty$ to obtain

$$
V(c(t))=V(c(\tau))-\int_{\tau}^{t} D(c(s)) d s
$$

By Theorem 5.1 of ref. 2, $c:[0, T) \rightarrow X^{+}$is continuous, and Lemma 4.2 of ref. 3 and (5.41) imply the continuity of $V: X^{+} \rightarrow \mathbb{R}$. Hence, passing to the limit $\tau \downarrow 0$ in (5.42) gives (5.5) and concludes the proof.

We now prove that equilibria of the type (5.1) are the only possible ones

Theorem 5.3. With the assumptions of Theorem 5.2 we have:

(i) Let $\rho<\infty$ and $0 \leqslant \rho \leqslant \rho_{s}$. Then there exists exactly one equilibrium $c^{\rho}$ of (1.1) with density $\rho$, and it is given by

$$
c_{j}^{\rho}=Q_{j}(z(\rho))^{j}, \quad j=1,2, \ldots
$$

where $z(\rho)$ is the unique positive solution of $F(z)=\rho$. 
(ii) Let $\rho \in\left(\rho_{s}, \infty\right)$. Then there is no equilibrium of (1.1) with density $\rho$.

Proof. The existence of such an equilibrium for $\rho \in\left[0, \rho_{s}\right]$ and its nonexistence for $\rho \in\left(\rho_{s}, \infty\right)$ follow from the definition of $\rho_{s}$ and the arguments in the beginning of this section.

The uniqueness in case (i) follows from the energy equation: if $c$ is an equilibrium of (1.1) with $\|c\|=\rho$, then, by Theorem 5.2,

$$
\int_{0}^{r} \sum_{j, k=1}^{\infty} W_{j, k}(c(s))\left[\log Q_{j+k} c_{j}(s) c_{k}(s)-\log Q_{j} Q_{k} c_{j+k}(s)\right] d s=0
$$

for all $t \in[0, T)$, and since all terms in the sum are nonnegative, $c$ must satisfy $W_{j, k}(c)=0$, or

$$
\frac{c_{j+k}}{Q_{j+k}}=\frac{c_{j}}{Q_{j}} \frac{c_{k}}{Q_{k}}
$$

By $\mathrm{H} 4.1$ these two conditions are equivalent. Hence we must have $c_{j}=Q_{j} z^{j}$ for some $z \geqslant 0$. As $\|c\|=\rho$, and $F(z)$ is strictly increasing for $z>0$, we obtain the existence of a unique $z=z(\rho)$ such that $\|c\|=\rho$.

\section{COAGULATION-FRAGMENTATION: ASYMPTOTIC BEHAVIOR OF SOLUTIONS}

\subsection{Preliminaries}

In order to handle the coagulation-fragmentation case, and since the hypotheses presently under consideration do not ensure uniqueness of solution, we must use the concept of generalized flows ${ }^{(2,3)}$ in the study of the asymptotic behavior of solutions to (1.1).

Definition 2. A generalized flow $\mathscr{G}$ on a metric space $Y$ is a family of continuous mappings $\phi:[0, \infty) \rightarrow Y$ such that:

(i) If $\phi \in \mathscr{G}$ and $\tau \geqslant 0$, then $\phi_{\tau} \in \mathscr{G}$, where $\phi_{r}(t)={ }^{\mathrm{der}} \phi(t+\tau)$, $\tau \in[0, \infty)$.

(ii) If $y \in Y$, there exists at least one $\phi \in \mathscr{G}$ with $\phi(0)=y$.

(iii) If $\phi_{j} \in \mathscr{G}$ with $\phi_{j}(0)$ convergent in $Y$ as $j \rightarrow \infty$, then there exists a subsequence $\phi_{j_{k}}$ of $\phi_{j}$ and a $\phi \in \mathscr{G}$ such that $\phi_{j_{k}}(t) \rightarrow \phi(t)$ in $Y$ uniformly for $t$ in compact intervals of $[0, \infty)$.

The following two results were proved in ref. 2 . 
Proposition 6.1. Assume (1.3). Let $\mathscr{G}$ denote the set of all densityconserving solutions of $(1.1)$ on $[0, \infty)$. Then $\mathscr{G}$ is a generalized flow on the closed metric subspace $X^{+}$of $X$.

Proposition 6.2. Let $g_{j} \geqslant 0$ with $g_{j}=o(j)$ as $j \rightarrow \infty$. Assume that $a_{j, k} \leqslant g_{j}+g_{k}$ and $b_{j, k} \leqslant g_{j} g_{k}$ for all $j, k$. For $\rho>0$ let $\mathscr{G}_{\rho}$ denote the set of all solutions of $(1.1)$ on $[0, \infty)$ with initial data in $B_{\rho}^{+}$. Then $\mathscr{G}_{\rho}$ is a generalized flow on $B_{\rho}^{+}$.

Let $\mathscr{G}$ be a generalized flow on a metric space $Y$. For $\phi \in \mathscr{G}$ the positive orbit of $\phi$ is defined by $\mathcal{O}^{+}(\phi)=\bigcup_{1 \geqslant 0} \phi(t)$, and the $\omega$-limit set of $\phi$ is the set $\omega(\phi)=\left\{y \in Y \mid\right.$ there exists a sequence $t_{j} \rightarrow \infty$ such that $\left.\phi\left(t_{j}\right) \rightarrow y\right\}$. The following result gives conditions under which $\omega(\phi)$ is nonempty (see ref. 3 for a proof):

Proposition 6.3. Let $\mathscr{G}$ be a generalized flow on $Y$. Let $\phi \in \mathscr{G}$ and suppose $\mathcal{O}^{+}(\phi)$ is relatively compact. Then $\omega(\phi)$ is nonempty and invariant, and $\operatorname{dist}(\phi(t), \omega(\phi)) \rightarrow 0$. Furthermore, if $\mathscr{V}: Y \rightarrow \mathbf{R}$ is a continuous Lyapunov function on $Y$, then $\omega(\phi)$ consists of complete orbits along which $\mathscr{V}$ has the constant value $\mathscr{V}^{\infty}=\lim _{t \rightarrow \infty} \mathscr{V}(\phi(t))$.

\subsection{Stabilization of Solutions: Weak Convergence Results}

We now apply the results of the previous paragraph and of Section 5 to get convergence of solutions to (1.1) to a single equilibrium as $t \rightarrow \infty$. The proof is the same as in ref. 3 for the Becker-Döring case, and it is reproduced here for completeness.

Theorem 6.4. Assume (1.7), $\mathrm{H} 1-\mathrm{H} 4$, and $b_{j, k} \leqslant o(j) o(k)$ as $|(j, k)| \rightarrow \infty$. Suppose $Q_{j}^{1 / j} \rightarrow z_{s}^{-1}$ as $j \rightarrow \infty$, and $0<z_{s}<\infty$.

Let $c$ be a solution of (1.1) with initial data $c(0)=c_{0},\left\|c_{0}\right\|=\rho_{0}$, and let $c^{\rho}$ be defined by $(5.1)$ with $\left\|c^{\rho}\right\|=\rho$.

Then $c(t) \stackrel{\dot{\Delta}}{c^{\rho}}$ as $t \rightarrow \infty$ for some $\rho$ satisfying $0 \leqslant \rho \leqslant \min \left\{\rho_{0}, \rho_{s}\right\}$.

Proof. If $\rho_{0}=0$, then $\rho(t) \equiv 0$ and thus $c(t) \equiv 0=c^{0}$. Suppose $\rho_{0}>0$. By Proposition $6.2, \mathscr{G}_{\rho_{0}}$ is a generalized flow on $B_{p_{0}}^{+}$. By Proposition 4.5 of ref. $3, V_{z s}$ is continuous on $B_{\rho_{0}}^{+}$and by Theorem 5.2

$$
V_{z_{s}}(c(t))=V_{z_{s}}\left(c_{0}\right)-\int_{0}^{t} D(c(s)) d s, \quad t \geqslant 0
$$

Since $\rho(t)$ is bounded, $\mathcal{O}^{+}(c)$ is relatively compact in $B_{\rho_{0}}^{+}$and thus, by Proposition 6.3, $\omega(c)$ is nonempty and consists of solutions $c(\cdot)$ along which $V_{z_{s}}$ has the constant value

$$
V_{z_{s}}^{\infty}=\lim _{t \rightarrow \infty} V_{z_{s}}(c(t))
$$


Let $\bar{c}(\cdot)$ be a nonzero solution in $\omega(c)$. Clearly $0 \leqslant\|\bar{c}(\cdot)\| \leqslant \rho_{0}$. Applying (6.1) to $\bar{c}(\cdot)$, we obtain

$$
\int_{0}^{t} D(\bar{c}(s)) d s=0 \quad \text { for all } \quad t \geqslant 0
$$

and necessarily

$$
\bar{c}_{j}(t)=Q_{j}\left(c_{1}(t)\right)^{j} \quad \text { for al } j \geqslant 1 \text { and } t \geqslant 0
$$

By density conservation, we have, for all $t \geqslant 0$,

$$
\sum_{j=1}^{\infty} j \bar{c}_{j}(t)=\sum_{j=1}^{\infty} j Q_{j}\left(\bar{c}_{1}(t)\right)^{j}=\sum_{j=1}^{\infty} j Q_{j}\left(\bar{c}_{1}(0)\right)^{j}
$$

so $\bar{c}_{1}(t)$ must be a constant, hence $\bar{c}$ must be an equilibrium. Consequently $\omega(c)$ consists of equilibria $c^{\rho}$ and we must have $\rho \leqslant \rho_{s}$. Thus $0 \leqslant \rho \leqslant$ $\min \left\{\rho_{0}, \rho_{s}\right\}$ and $V_{z_{s}}\left(c^{\rho}\right)=V_{z_{s}}^{\infty}$. Since, by Proposition 4.3 of ref. $3, V_{z_{s}}\left(c^{\rho}\right)$ is strictly decreasing in $\rho$, we must have $\omega(c)=\left\{c^{\rho}\right\}$ for a unique $\rho$ in $\left[0, \min \left\{\rho_{0}, \rho_{s}\right\}\right]$, and, by Proposition 6.3, $\operatorname{dist}\left(c(t), c^{\rho}\right) \rightarrow 0$ as $t \rightarrow \infty$, where $\operatorname{dist}(\cdot, \cdot)$ is the metric of $B_{\rho_{0}}^{+}$, which implies $c(t) \stackrel{\dot{\sim}}{\sim} c^{\rho}$ as $t \rightarrow \infty$.

\subsection{Stabilization of Solutions: Strong Convergence Results for the Generalized Becker-Döring Equations}

As discussed in the introduction, the identification of the density $\rho$ of the $\omega$-limit solution in the previous section has not been accomplished in full generality. Consequently, it is not yet possible to decide if, or under what circumstances, the weak* convergence to equilibrium in Theorem 6.4 in indeed a weak* convergence (i.e., $\rho<\rho_{0}$ ) or is actually a strong convergence $\left(\rho=\rho_{0}\right)$. Since this problem for the Becker-Döring equations was solved under conditions that are a particular case of the ones now under consideration, ${ }^{(1,3)}$ we expect results like Theorem 5 of ref. 1 or Theorem 5.6 of ref. 3 still to be true for the present case.

In this subsection we prove a result for a particular case which is obtained by setting $a_{j, k}=b_{j, k}=0$ if $\min \{j, k\}>N$ for some fixed $N \geqslant 2$. We shall call this case the "generalized-Becker-Döring equation," as the Becker-Döring system is recovered by setting $N=1$. Although more general than the Becker-Döring equation, this system is still considerably simpler than the general case obtained, formally, by setting $N=\infty$. The proof of the main result of this subsection, Theorem 6.5 , depends crucially of the fact that $N$ is a fixed positive integer, and so is not applicable to the general equations (1.1). 
Assume from now on:

H5. There exists a positive integer $N \geqslant 2$ such that

$$
a_{j, k}=b_{j, k}=0 \quad \text { if and only if } \min \{j, k\}>N
$$

Under this condition the coagulation-fragmentation equations (1.1) become the following generalized Becker-Döring system:

$$
\begin{cases}\dot{c}_{1}=-\sum_{k=1}^{\infty} W_{1, k}(c) & \\ \dot{c}_{j}=\frac{1}{2} \sum_{k=1}^{j-1} W_{j-k, k}(c)-\sum_{k=1}^{\infty} W_{j, k}(c), & 2 \leqslant j \leqslant N \\ \dot{c}_{j}=\frac{1}{2} \sum_{k=1}^{j-1} W_{j-k, k}(c)-\sum_{k=1}^{N} W_{j, k}(c), & N+1 \leqslant j \leqslant 2 N \\ \dot{c}_{j}=\frac{1}{2} \sum_{k=1}^{N} W_{j-k, k}(c)-\sum_{k=1}^{N} W_{j, k}(c), & j \geqslant 2 N+1\end{cases}
$$

Since it is a special case of (1.1), all previous results still hold for this system under the same assumptions. Furthermore, we have the following "phase transition" behavior.

Theorem 6.5. In addition to the hypotheses of Theorem 6.4, assume $\mathrm{H} 5$ and suppose there exists a constant $M>0$ and a positive integer $k_{0}$ such that, for all $l=1, \ldots, N$ and all $i>k_{0}$,

$$
\begin{aligned}
& 0 \leqslant b_{i, 1}-a_{i, l} Q_{i} z_{s}^{l} \leqslant M \\
& 0 \leqslant b_{i-1,1}-a_{i, 1} Q_{l} z_{s}^{l}
\end{aligned}
$$

Let $\rho_{s}<\infty$, and suppose $c_{0} \in X^{+}$satisfies $\sum_{j=1}^{\infty} c_{0 j} /\left(Q_{j} z_{s}^{j}\right)<\infty$. Suppose also that $c$ is the only solution of $(6.3)$ on $[0, \infty)$ with $c(0)=c_{0}$. Then:

(i) If $0 \leqslant \rho_{0} \leqslant \rho_{s}$, then $c(t) \rightarrow c^{\rho_{0}}$ strongly in $X$ as $t \rightarrow \infty$, and

$$
\lim _{t \rightarrow \infty} V(c(t))=V\left(c^{\rho_{0}}\right)
$$

(ii) If $\rho_{0}>\rho_{s}$, then $c(t) \stackrel{\dot{\sim}}{\rho^{\rho_{s}}}$ as $t \rightarrow \infty$ and

$$
\lim _{t \rightarrow \infty} V(c(t))=V\left(c^{\rho_{s}}\right)+\left(\rho_{0}-\rho_{s}\right) \log z_{s}
$$

Remark 6.6. It is presently not known if the assumptions of the theorem ensure uniqueness of solution. However, for certain choices of parameters uniqueness can be guaranteed. For example, if $0 \leqslant \alpha \leqslant 1 / 2$ in 
$\mathrm{H} 1$ and $b_{j, k} \leqslant K(j k)^{\beta}$ with $\beta \leqslant 1 / 2$, then, by Theorem 4.1 of ref. 2, solutions to (6.3) are unique. This condition is fulfilled by certain types of physically important coefficients, for which $\alpha, \beta \simeq 1 / 3$. $^{(2.3)}$

Remark 6.7. It is easy to check that the assumptions of Theorem 6.5 hold if $a_{j, k}=K(j+k)^{\nu}$ and $b_{j, k}=K(j+k)^{v} \exp \left\{\lambda\left[(j+k)^{\mu}-j^{\mu}-k^{\mu}\right]\right\}$ for $\min \{j, k\} \leqslant N$, and $a_{j, k}=b_{j, k}=0$ otherwise, where $K, \lambda, \mu$, and $v$ are positive constants and $0<\mu<1, v \leqslant 1-\mu$. For these coefficients the sequence $\left(Q_{j}\right)$ is given by $Q_{j}=\exp \left[\lambda\left(j-j^{\mu}\right)\right]$ and the condition on the initial data is $\sum_{j=1}^{\infty} \exp \left(\lambda j^{\mu}\right) c_{0 j}<\infty$.

We need some preliminary lemmas on the behavior of moments of the type $\sum_{j=1}^{\infty} c_{j}(t) /\left(Q_{j} z_{s}^{j}\right)$ before proving the above theorem.

Lemma 6.8. Assume (1.3), (1.7), (6.4), and H5. Let $\mathscr{K}, \mathscr{M}$ be constants such that

$$
\mathscr{K} \geqslant \mathscr{M} \geqslant \max _{1 \leqslant j \leqslant N}\left(Q_{j} z_{s}^{j}\right)^{-1}+\max _{\substack{1 \leqslant j \leqslant N \\ 1 \leqslant k \leqslant k_{0}}} \frac{\left|b_{j, k}-a_{j, k} Q_{j} z_{s}^{j}\right|}{a_{j, k} Q_{j} Q_{k} z_{s}^{j+k}}
$$

Define

$$
g_{j}=\mathscr{K} j+\frac{1}{Q_{j} z_{s}^{j}}-\mathscr{M} \quad \text { for } \quad j=1,2, \ldots
$$

Then:

(i) $g_{j}>0$ for all $j \geqslant 1$.

(ii) $g_{j+k}-g_{j}-g_{k} \geqslant 0$ for all $1 \leqslant j \leqslant N$ and $k \geqslant 1$.

(iii) $\left(g_{j+k}-g_{k}\right) a_{j, k} \sim \mathcal{O}\left(g_{k}\right)$ as $k \rightarrow \infty$ for all $1 \leqslant j \leqslant N$.

Proof. (i) $g_{j}=\mathscr{K} j+1 / Q_{j} z_{s}^{j}-\mathscr{M}>\mathscr{K} j-\mathscr{M} \geqslant \mathscr{M}(j-1) \geqslant 0$.

(ii) Let $k>k_{0}$. Then

$$
\begin{aligned}
g_{j+k}-g_{j}-g_{k} & =\frac{1}{Q_{j+k} z_{s}^{j+k}}-\frac{1}{Q_{j} z_{s}^{j}}-\frac{1}{Q_{k} z_{s}^{k}}+\mathscr{M} \\
& =\frac{1}{a_{j, k} Q_{j} z_{s}^{j} Q_{k} z_{s}^{k}}\left(b_{j, k}-a_{j, k} Q_{j} z_{s}^{j}-a_{j, k} Q_{k} z_{s}^{k}\right)+\mathscr{M} \\
& \geqslant-\frac{1}{Q_{j} z_{s}^{j}}+\mathscr{M} \geqslant 0
\end{aligned}
$$


If $1 \leqslant k \leqslant k_{0}$, then

$$
\begin{aligned}
g_{j+k}-g_{j}-g_{k} & =\frac{b_{j, k}-a_{j, k} Q_{j} z_{s}^{j}}{a_{j, k} Q_{j} Q_{k} z_{s}^{j+k}}-\frac{1}{Q_{j} z_{s}^{j}}+\mathscr{M} \\
& \geqslant-\left(\frac{\left|b_{j, k}-a_{j, k} Q_{j} z_{s}^{j}\right|}{a_{j, k} Q_{j} Q_{k} z_{s}^{j+k}}+\frac{1}{Q_{j} z_{s}^{j}}\right)+\mathscr{M} \\
& \geqslant-\left(\max _{\substack{1 \leqslant j \leqslant N \\
1 \leqslant k \leqslant k_{0}}} \frac{\left|b_{j, k}-a_{j, k} Q_{j} z_{s}^{j}\right|}{a_{j, k} Q_{j} Q_{k} z_{s}^{j+k}}+\max _{1 \leqslant j \leqslant N}\left(Q_{j} z_{s}^{j}\right)^{-1}\right)+\mathscr{M} \geqslant 0
\end{aligned}
$$

(iii) Since we are interested in the case $k \rightarrow \infty$, we can assume $k>k_{0}$. Then

$$
\begin{aligned}
\left(g_{j+k}-g_{k}\right) a_{j, k} & =\left(\mathscr{K} j+\frac{1}{Q_{j+k} z_{s}^{j+k}}-\frac{1}{Q_{k} z_{s}^{k}}\right) a_{j, k} \\
& \leqslant K_{a} \mathscr{K} j(j+k)+\frac{1}{Q_{k} z_{s}^{k}} \frac{b_{j, k}-a_{j, k} Q_{j} z_{s}^{j}}{Q_{j} z_{s}^{j}} \\
& \leqslant K_{a} \mathscr{K} N^{2}+K_{a} \mathscr{K} N k+M \mathscr{M} \frac{1}{Q_{k} z_{s}^{k}} \\
& \leqslant \text { const } \cdot\left[g_{k}+\mathscr{M}\right]
\end{aligned}
$$

provided we choose the constant sufficiently large. Since $g_{k} \rightarrow \infty$ as $k \rightarrow \infty$, the result follows.

Lemma 6.9. With the assumptions of the previous lemma, suppose $c_{0} \in X^{+}$satisfy $\sum_{j=1}^{\infty} g_{j} c_{0 j}<\infty$. Let $c$ be an admissible solution of (6.3) on $[0, T)$ with $c(0)=c_{0}$.

Then $\sum_{j=1}^{\infty} g_{j} c_{j}(t)<\infty$ for all $t \in[0, T)$.

Proof. Let $c^{(n)}$ be a solution of the $n$-dimensional density-conserving truncation of (6.3) with initial data $c_{0}^{(n)}$. Then, for $n \geqslant 2 N+1$,

$$
\begin{aligned}
\sum_{j=1}^{n} g_{j} \dot{c}_{j}^{(n)} & =\frac{1}{2} \sum_{j=1}^{n-1} \sum_{k=1}^{n-j}\left(g_{j+k}-g_{j}-g_{k}\right) W_{j, k}\left(c^{(n)}\right) \\
& \leqslant \frac{1}{2} \sum_{j=1}^{n-1} \sum_{k=1}^{n-j}\left(g_{j+k}-g_{j}-g_{k}\right) a_{j, k} c_{j}^{(n)} c_{k}^{(n)} \\
& =\sum_{j=1}^{N} \sum_{k=j}^{n-j}\left(g_{j+k}-g_{j}-g_{k}\right) a_{j, k} c_{j}^{(n)} c_{k}^{(n)}-\frac{1}{2} \sum_{j=1}^{N}\left(g_{2 j}-2 g_{j}\right) a_{j, j}\left(c_{j}^{(n)}\right)^{2}
\end{aligned}
$$




$$
\begin{aligned}
& \leqslant \sum_{j=1}^{N} \sum_{k=1}^{n}\left(g_{j+k}-g_{j}-g_{k}\right) a_{j, k} c_{j}^{(n)} c_{k}^{(n)} \\
& \leqslant \sum_{j=1}^{N} \sum_{k=1}^{n}\left(g_{j+k}-g_{k}\right) a_{j, k} c_{j}^{(n)} c_{k}^{(n)} \\
& \leqslant \text { const } \cdot \sum_{j=1}^{N} c_{j}^{(n)} \sum_{k=1}^{n} g_{k} c_{k}^{(n)} \\
& \leqslant \text { const } \cdot\left\|c_{0}\right\| \sum_{k=1}^{n} g_{k} c_{k}^{(n)}
\end{aligned}
$$

Hence, by Gronwall's inequality

$$
\sum_{j=1}^{n} g_{j} c_{j}^{(n)} \leqslant\left(\sum_{j=1}^{n} g_{j} c_{0 j}\right) e^{(\text {const }) t} \leqslant\left(\sum_{j=1}^{\infty} g_{j} c_{0 j}\right) e^{(\text {const }) t}
$$

and by the admissibility of $c$ we can pass to the limit in this inequality to obtain the result.

Lemma 6.10 With the assumptions of Lemma 6.9, the function $t \mapsto \sum_{j=1}^{\infty} g_{j} c_{j}(t)$ is continuous on $[0, T)$.

Proof. Let $c^{(n)}$ be a solution of the $n$-dimensional maximal truncation of (6.3) with initial data $c_{0}^{(n)}$. Then, for all $0 \leqslant t_{1}<t_{2}<\infty$ and all $n$,

$$
\begin{aligned}
& \left|\sum_{j=1}^{\infty} g_{j} c_{j}\left(t_{2}\right)-\sum_{j=1}^{\infty} g_{j} c_{j}\left(t_{1}\right)\right| \\
& \leqslant\left|\sum_{j=1}^{\infty} g_{j}\left(c_{j}\left(t_{2}\right)-c_{j}^{(n)}\left(t_{2}\right)\right)\right|+\left|\sum_{j=1}^{\infty} g_{j}\left(c_{j}\left(t_{1}\right)-c_{j}^{(n)}\left(t_{1}\right)\right)\right| \\
& \quad+\left|\sum_{j=1}^{n} g_{j}\left(c_{j}^{(n)}\left(t_{2}\right)-c_{j}^{(n)}\left(t_{1}\right)\right)\right|
\end{aligned}
$$

By admissibility of $c$ there exists a sequence $n_{k} \rightarrow \infty$ such that $c_{j}^{\left(m_{k}\right)} \rightarrow c_{j}$ for all $j$ as $k \rightarrow \infty$ and by density conservation we have $c^{\left(n_{k}\right)} \rightarrow c$ in $X^{+}$as $k \rightarrow \infty$. Consider $n$ to be an arbitrary element of $\left(n_{k}\right)$. By Lemma 6.9 the first two terms above can be made arbitrarily small by choosing $n$ sufficiently large, with $t_{1}$ and $t_{2}$ kept fixed. To obtain an estimate for the last term, we are going to derive a lower bound for the rate of change of $\sum_{j=1}^{n} g_{j} c_{j}^{(n)}$. Let $n \geqslant 2 N+1$, 


$$
\begin{aligned}
& \sum_{j=1}^{n} g_{j} \dot{c}_{j}^{(n)}=\frac{1}{2} \sum_{j=1}^{n-1} \sum_{k=1}^{n-j}\left(g_{j+k}-g_{j}-g_{k}\right) W_{j, k}\left(c^{(n)}\right) \\
& \geqslant-\frac{1}{2} \sum_{j=1}^{n-1} \sum_{k=1}^{n-j}\left(g_{j+k}-g_{j}-g_{k}\right) b_{j, k} c_{j+k}^{(n)} \\
& \geqslant-\sum_{j=1}^{N} \sum_{k=1}^{n-j}\left(g_{j+k}-g_{j}-g_{k}\right) b_{j, k} c_{j+k}^{(n)} \\
& =-\sum_{j=1}^{N} \sum_{k=1}^{n-j}\left(\frac{b_{j, k}-a_{j, k} Q_{j} z_{s}^{j}-a_{j, k} Q_{k} z_{s}^{k}}{a_{j, k} Q_{k} z_{s}^{k} Q_{j} z_{s}^{j}}+\mathscr{M}\right) b_{j, k} c_{j+k}^{(n)} \\
& =-\left(\sum_{j=1}^{N} \sum_{k=1}^{n-j} \frac{b_{j, k}-a_{j, k} Q_{j} z_{s}^{j}}{Q_{j} z_{s}^{j}} \frac{b_{j, k}}{a_{j, k} Q_{k} z_{s}^{k}} c_{j+k}^{(n)}\right. \\
& \left.-\sum_{j=1}^{N} \sum_{k=1}^{n-j} \frac{1}{Q_{j} z_{s}^{j}} b_{j . k} c_{j+k}^{(n)}+\mathscr{M} \sum_{j=1}^{N} \sum_{k=1}^{n-j} b_{j . k} c_{j+k}^{(n)}\right) \\
& \geqslant-\left(\sum_{j=1}^{N} \sum_{k=1}^{n-j} \frac{b_{j, k}-a_{j, k} Q_{j} z_{s}^{j}}{Q_{j} z_{s}^{j}} \frac{b_{j, k}}{a_{j, k} Q_{k} z_{s}^{k}} c_{j+k}^{(n)}\right. \\
& \left.+\mathscr{M} \sum_{j=1}^{N} \sum_{k=1}^{n-j} b_{j, k} c_{j+k}^{(n)}\right)
\end{aligned}
$$

Without loss of generality we can choose $M$ so large that the right-hand inequality in (6.4) is valid for all $i \geqslant 1$ and so we can bound the first double sum in $(6.6)$ by

$$
\begin{aligned}
& \sum_{j=1}^{N} \sum_{k=1}^{n-j} \frac{b_{j, k}-a_{j, k} Q_{j} z_{s}^{j}}{Q_{j} z_{s}^{j}} \frac{b_{j, k}}{a_{j, k} Q_{k} z_{s}^{k}} c_{j+k}^{(n)} \\
& \quad \leqslant M \mathscr{M} \sum_{j=1}^{N} \sum_{k=1}^{n-j} \frac{b_{j, k}}{a_{j, k} Q_{k} z_{s}^{k}} c_{j+k}^{(n)} \\
& \quad=M \mathscr{M} \sum_{j=1}^{N} \sum_{p=j+1}^{n} \frac{b_{j, p-j}}{a_{j, p-j} Q_{p-j} z_{s}^{p-j}} c_{p}^{(n)} \\
& \quad=M \mathscr{M} \sum_{j=1}^{N} \sum_{p=j+1}^{n} \frac{Q_{j} z_{s}^{j}}{Q_{p} z_{s}^{p}} c_{p}^{(n)} \\
& \quad=M \mathscr{M} \sum_{j=1}^{N} Q_{j} z_{s}^{j} \sum_{p=j+1}^{n} \frac{1}{Q_{p} z_{s}^{p}} c_{p}^{(n)} \\
& \leqslant \text { const } \cdot \sum_{p=1}^{n} g_{p} c_{p}^{(n)}
\end{aligned}
$$


For the second double sum in (6.6) we have

$$
\begin{aligned}
\sum_{j=1}^{N} \sum_{k=1}^{n-j} b_{j, k} c_{j+k}^{(n)} & =\sum_{j=1}^{N} \sum_{p=j+1}^{n} b_{j, p-j} c_{p}^{(n)} \\
& \leqslant \text { const } \cdot \sum_{j=1}^{N} \sum_{p=j+1}^{n} j(p-j) c_{p}^{(n)} \\
& \leqslant \text { const } \cdot \sum_{j=1}^{N} j \sum_{p=j+1}^{n} p c_{p}^{(n)} \\
& \leqslant \text { const } \cdot \sum_{p=1}^{n} p c_{p}^{(n)} \\
& \leqslant \text { const } \cdot \sum_{p=1}^{n} g_{p} c_{p}^{(n)}
\end{aligned}
$$

Hence, we obtain

$$
\sum_{j=1}^{n} g_{j} \dot{c}_{j}^{(n)} \geqslant- \text { const } \cdot \sum_{j=1}^{n} g_{j} c_{j}^{(n)}
$$

with const a constant independent of $n$. This inequality, together with the one in the proof of Lemma 6.9, gives, for $t_{1}, t_{2} \in \mathscr{I}$, a compact set of $[0, \infty)$,

$$
\left|\sum_{j=1}^{n} g_{j}\left(c_{j}^{(n)}\left(t_{2}\right)-c_{j}^{(n)}\left(t_{1}\right)\right)\right| \leqslant K_{g}\left|t_{2}-t_{1}\right|
$$

where $K_{s}$ is a constant independent of $n$.

Lemma 6.11. With the assumptions of Lemma 6.10, the series $\sum_{j} g_{j} c_{j}(t)$ is uniformly convergent on compact sets of $[0, T)$.

Proof. Defining

$$
f_{n}(t)=\sum_{j=1}^{n} g_{j} c_{j}(t)
$$

we have that $f_{n}$ is continuous, $f_{n+1} \geqslant f_{n}$, and by Lemmas 6.9 and 6.10 , $\lim _{n \rightarrow \infty} f_{n}(t)$ exists for all $t \geqslant 0$ and is a continuous function. Hence, by Dini's theorem, the series converges uniformly for $t$ in compact sets of $[0, T)$.

Proof of Theorem 6.5. We will prove that if $c(t) \doteq c^{\rho}$ as $t \rightarrow \infty$ for some $\rho<\rho_{s}$, then $c(t) \rightarrow c^{\rho}$ strongly, so that, by density conservation, $\rho=\rho_{0}=\left\|c_{0}\right\|$. 
Hence, for $\rho_{0}<\rho_{s}$, Theorem 6.4 implies $0 \leqslant \rho \leqslant \rho_{0}<\rho_{s}$ and using strong convergence and density conservation, $\rho=\rho_{0}$. For $\rho_{0}>\rho_{s}$, then $0 \leqslant \rho \leqslant \rho_{s}$. If $\rho<\rho_{s}$, then we would have strong convergence in $X$ to $c^{\rho}$, which, by density conservation, would imply $\rho_{0}=\rho<\rho_{s}$, contradicting the original assumption $\rho_{0}>\rho_{s}$. So if $\rho_{0}>\rho_{s}$, we have $\rho=\rho_{s}$. Similarly, if $\rho_{0}=\rho_{s}$, density conservation and the strong convergence result above imply $\rho=\rho_{0}$. The results on the limit behavior of the Lyapunov function follow from Theorem 4.4 of ref. 3 .

So we are left to prove that, if $c(t) \doteq c^{p}$ in $X$ as $t \rightarrow \infty$ for some $\rho<\rho_{s}$, then $c(t) \rightarrow c^{\rho}$ strongly in $X$ as $t \rightarrow \infty$.

By Lemma 6.9 we have $\sum_{j=1}^{\infty} c_{j}(t) /\left(Q_{j} z_{s}^{j}\right)<\infty$ for all $t \geqslant 0$, and by Lemma 6.11 this series is uniformly convergent for $t$ in compact intervals of $[0, \infty)$. Define

$$
x_{j}(t) \stackrel{\text { def }}{=} \frac{c_{j}(t)}{Q_{j} z_{s}^{j}}
$$

Since $c(t) \doteq c^{\rho}$ with $\rho<\rho_{s}$ as $t \rightarrow \infty$, we have, for fixed $r_{0} \geqslant 1$, the existence of a $t_{1}$ such that for all $t>t_{1}$ and all $1 \leqslant j \leqslant r_{0}, x_{j}(t)<1$. We are going to prove that $x_{j}(t)<K_{1}+1$, where $K_{1}=\sup \left\{x_{j}\left(t_{1}\right): j \geqslant 1\right\}$. If not, by the uniform convergence of $\sum_{j=1}^{\infty} x_{j}(t)$, there exist a $t_{2} \geqslant t_{1}$ and a minimal $n>r_{0}$ such that $x_{n}\left(t_{2}\right)=K_{1}+1 \geqslant x_{r}\left(t_{2}\right)$ for $r \neq n$ and $x_{n}(t) \leqslant x_{n}\left(t_{2}\right)$ for $t \in\left[t_{1}, t_{2}\right]$. Now, taking $r_{0} \geqslant \max \left\{2 N, k_{0}\right\}$, using the definition of $x_{n}$ and the detailed balance condition, we obtain

$$
\begin{aligned}
\dot{x}_{n}= & \frac{1}{Q_{n} z_{s}^{n}}\left(\sum_{k=1}^{N} W_{n-k, k}-\sum_{k=1}^{N} W_{n, k}\right) \\
= & \frac{1}{Q_{n} z_{s}^{n}} \sum_{k=1}^{N}\left(a_{n-k, k} x_{n-k} x_{k} Q_{n-k} z_{s}^{n-k} Q_{k} z_{s}^{k}-b_{n-k, k} x_{n} Q_{n} z_{s}^{n}\right. \\
& \left.-a_{n-k, k} x_{n} x_{k} Q_{n} z_{s}^{n} Q_{k} z_{s}^{k}+b_{n, k} x_{n+k} Q_{n+k} z_{s}^{n+k}\right) \\
= & \sum_{k=1}^{N}\left(b_{n-k, k} x_{n-k} x_{k}-b_{n-k, k} x_{n}-a_{n, k} x_{n} x_{k} Q_{k} z_{s}^{k}+a_{n, k} x_{n+k} Q_{k} z_{s}^{k}\right) \\
= & \sum_{k=1}^{N}\left[\left(x_{k}-1\right)\left(b_{n-k, k}-a_{n, k} Q_{k} z_{s}^{k}\right) x_{n}\right. \\
& \left.+b_{n-k, k}\left(x_{n-k}-x_{n}\right) x_{k}+a_{n, k} Q_{k} z_{s}^{k}\left(x_{n+k}-x_{n}\right)\right]
\end{aligned}
$$

where (6.8) is obtained from (6.7) by adding and subtracting $\sum_{k=1}^{N}\left(b_{n-k, k} x_{n} x_{k}+a_{n, k} Q_{k} z_{s}^{k} x_{n}\right)$ and rearranging the terms. By (6.5), the minimality of $n$, the positivity of solutions, and the fact that $x_{j}(t)<1$ for 
$1 \leqslant j \leqslant r_{0}, t>t_{1}$, we obtain $\dot{x}_{n}\left(t_{2}\right)<0$, which contradicts $x_{n}(t) \leqslant x_{n}\left(t_{2}\right)$ for $t_{1} \leqslant t \leqslant t_{2}$. So we have $c_{j}(t) \leqslant\left(K_{1}+1\right) Q_{j} z_{s}^{j}$ for all $t \geqslant t_{1}, j \geqslant 1$. Now, since $\sum_{j=1}^{\infty} j Q_{j} z_{s}^{j}<\infty$, we have $\gamma_{c}^{+}$relatively compact in $X^{+}$and so $c(t) \rightarrow c^{\rho}$ strongly, as $t \rightarrow \infty$.

Theorem 6.12. Let $\rho_{s}=\infty$, and assume the remaining hypotheses of Theorem 6.5 are still valid, with $z_{s}$ changed to a $\tilde{z} \in\left(0, z_{s}\right)$.

With $c$ an admissible solution of $(6.3)$ on $[0, \infty)$ with initial data $c_{0}$, we have $c(t) \rightarrow c^{\rho_{0}}$ strongly in $X$ as $t \rightarrow \infty$.

Proof. In this case $\rho_{s}=\lim _{z \dagger z_{s}} F(z)=\infty$ and so we cannot use the bound $c_{j}(t) \leqslant\left(K_{1}+1\right) Q_{j} z_{s}^{j}$ to prove compacity of $\mathcal{O}^{+}(c)$. However, since $\rho_{s}=\infty$ and $\rho_{0}=\left\|c_{0}\right\|<\infty$ there exists a $0<\tilde{z}<z_{s}$ such that $\rho_{0}<F(\tilde{z})<\infty$. The proof of Theorem 6.5 is now applicable with $\tilde{z}$ instead of $z_{s}$.

\section{ACKNOWLEDGMENT}

The work of F. P. d. C. was supported by grant 9/90/B from Fundação Calouste Gulbenkian.

\section{REFERENCES}

1. J. Ball and J. Carr, Asymptotic behaviour of solutions to the Becker-Döring equation for arbitrary initial data, Proc. R. Soc. Edinburgh 108A:109-116 (1988).

2. J. Ball and J. Carr, The discrete coagulation-fragmentation equations: Existence, uniqueness, and density conservation, J. Stat. Phys. 61:203-234 (1990).

3. J. Ball, J. Carr, and O. Penrose, The Becker-Döring cluster equations: Basic properties and asymptotic behaviour of solutions, Commun. Math. Phys. 104:657-692 (1986).

4. J. Carr, Asymptotic behaviour of solutions to the coagulation-fragmentation equations. I. The strong fragmentation case, Proc. $R$. Soc. Edinburgh 121 A:231-244 (1992).

5. J. Carr and F. P. Costa, Instantaneous gelation in coagulation dynamics, Z. Angew. Math. Phys. 43:974-983 (1992).

6. M. Slemrod, Trend to equilibrium in the Becker-Döring cluster equations, Nonlinearity 2:429-443 (1989).

7. M. Shirvani and H. van Roessel, The mass-conserving solutions of Smoluchowski's coagulation equation: The general bilinear kernel, $Z$. Angew. Math. Phys. 43:526-535 (1992). 